\title{
Dye-loaded mesoporous polydopamine nanoparticles for multimodal tumor theranostics with enhanced immunogenic cell death
}

\author{
Ying Tian ${ }^{1,2 \dagger}$, Muhammad Rizwan Younis ${ }^{3+}$, Yuxia Tang ${ }^{1,2 \dagger}$, Xiang Liao ${ }^{1,2}$, Gang He ${ }^{3}$, Shouju Wang ${ }^{1,2}$,
} Zhaogang Teng ${ }^{4}$, Peng Huang ${ }^{3^{*}}$ (D) Longjiang Zhang ${ }^{1,2^{*}}$ and Guangming Lu $^{1,2^{*}}$

\begin{abstract}
Background: Tumor phototherapy especially photodynamic therapy (PDT) or photothermal therapy (PTT), has been considered as an attractive strategy to elicit significant immunogenic cell death (ICD) at an optimal tumor retention of PDT/PTT agents. Heptamethine cyanine dye (IR-780), a promising PDT/PTT agent, which can be used for near-infrared (NIR) fluorescence/photoacoustic (PA) imaging guided tumor phototherapy, however, the strong hydrophobicity, short circulation time, and potential toxicity in vivo hinder its biomedical applications. To address this challenge, we developed mesoporous polydopamine nanoparticles (MPDA) with excellent biocompatibility, PTT efficacy, and PA imaging ability, facilitating an efficient loading and protection of hydrophobic IR-780.

Results: The IR-780 loaded MPDA (IR-780@MPDA) exhibited high loading capacity of IR-780 (49.7 wt\%), good physiological solubility and stability, and reduced toxicity. In vivo NIR fluorescence and PA imaging revealed high tumor accumulation of IR-780@MPDA. Furthermore, the combined PDT/PTT of IR-780@MPDA could induce ICD, triggered immunotherapeutic response to breast tumor by the activation of cytotoxic T cells, resulting in significant suppression of tumor growth in vivo.
\end{abstract}

Conclusion: This study demonstrated that the as-developed compact and biocompatible platform could induce combined PDT/PTT and accelerate immune activation via excellent tumor accumulation ability, offering multimodal tumor theranostics with negligible systemic toxicity.

Keywords: Mesoporous polydopamine nanoparticles, IR-780, Photodynamic therapy, Photothermal therapy, Immunogenic cell death

\footnotetext{
*Correspondence: peng.huang@szu.edu.cn; kevinzhlj@163.com;

cjr.luguangming@vip.163.com

†Ying Tian, Muhammad Rizwan Younis and Yuxia Tang contributed equally to this work

${ }^{1}$ Department of Medical Imaging, Jinling Hospital, Medical School of Nanjing University, Nanjing 210002, People's Republic of China

${ }^{3}$ Marshall Laboratory of Biomedical Engineering, International Cancer Center, Laboratory of Evolutionary Theranostics (LET), School of Biomedical Engineering, Shenzhen University Health Science Center, Shenzhen 518060, People's Republic of China

Full list of author information is available at the end of the article
} permits use, sharing, adaptation, distribution and reproduction in any medium or format, as long as you give appropriate credit to the original author(s) and the source, provide a link to the Creative Commons licence, and indicate if changes were made. The images or other third party material in this article are included in the article's Creative Commons licence, unless indicated otherwise in a credit line to the material. If material is not included in the article's Creative Commons licence and your intended use is not permitted by statutory regulation or exceeds the permitted use, you will need to obtain permission directly from the copyright holder. To view a copy of this licence, visit http://creativecommons.org/licenses/by/4.0/. The Creative Commons Public Domain Dedication waiver (http://creativeco mmons.org/publicdomain/zero/1.0/) applies to the data made available in this article, unless otherwise stated in a credit line to the data. 


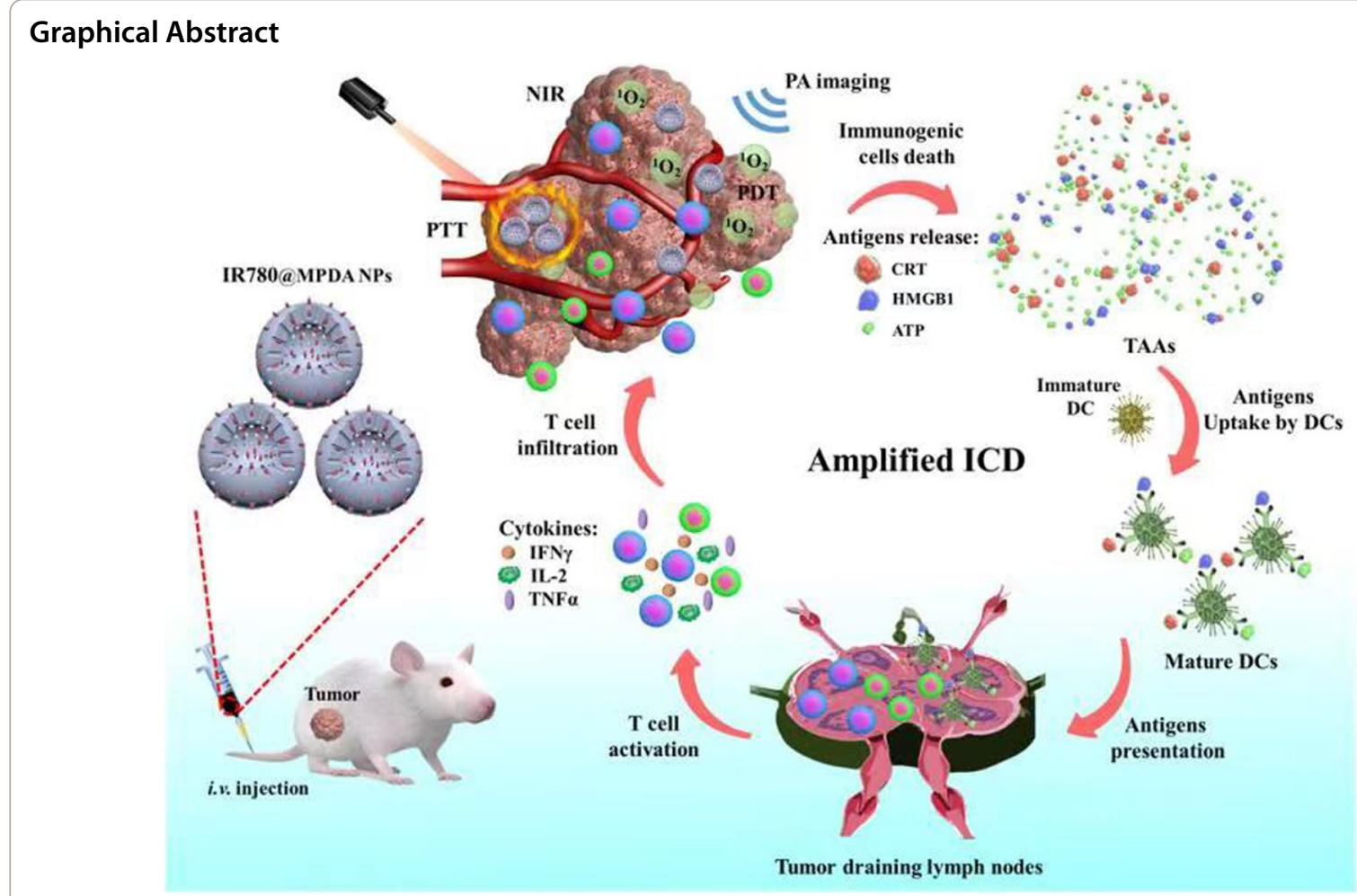

\section{Introduction}

Several anti-tumor nanomedicine strategies have been successfully developed, and among those, phototherapeutic tumor killing by photothermal therapy (PTT) or photodynamic therapy (PDT) has attracted significant attention [1-7]. PTT is an emerging phototherapeutic modality, which triggers photothermal tumor destruction due to localized hyperthermia generated by photoconversion nanoparticles under near-infrared (NIR) light irradiation. While, in PDT, photosensitizers (PSs) generate toxic reactive oxygen species (ROS) under laser irradiation to induce photodynamic tumor killing $[8,9]$. Recently, it has been demonstrated that both PTT and PDT can trigger significant immunogenic cell death (ICD) via producing or exposing tumor-associated agents from dead tumor cell residues [10-12]. Tumor cells undergoing ICD can evoke danger-associated molecular patterns (DAMPs) to recruit intratumoral T lymphocytes infiltration as well as an increased ratio of cytotoxic $\mathrm{T}$ lymphocytes (CTLs), resulting in the enhanced immune response [13-15]. Therefore, the development of agents with simultaneous PDT and PTT effects is advantageous to trigger significant ICD with better therapeutic efficacy $[16,17]$.

Heptamethine cyanine dye (IR-780) with a characteristic absorption peak at $780 \mathrm{~nm}$, has been applied in NIR fluorescence or PA imaging $[18,19]$. Moreover, because of the excellent light to heat conversion efficiency and ROS production capacity upon NIR laser irradiation, IR-780 was utilized as a multifunctional agent to trigger both PTT and PDT, simultaneously [20-23]. The "Two in One" therapy mediated by IR-780 could trigger the sustained tumor cell killing even at a lower PDT efficacy in solid tumor due to the limited oxygen supply [24-26]. Unfortunately, owing to the strong hydrophobicity and short circulation time in vivo, the further biomedical applications of IR-780 are restricted [27-29]. So, it is highly desirable to develop an IR-780 delivery platform for an efficient tumor theranostics.

Herein, IR-780 delivery platform based on mesoporous polydopamine nanoparticles (MPDA) is developed. The hydrophobic IR-780 is loaded onto MPDA through $\pi-\pi$ stacking interaction (Additional file 1: Fig. S1). The asprepared IR-780 loaded MPDA (IR-780@MPDA) exhibited good photothermal conversion ability, and thus are utilized as photoacoustic (PA) contrast agent and photothermal agent for photoacoustic imaging-guided PTT [30-33]. Overall, IR-780@MPDA showed high loading capacity (49.7 wt\%), low off-target toxicity, and excellent biocompatibility. The tumor accumulation of IR-780@MPDA was distinctively traced by NIR fluorescence and PA imaging in vivo, respectively. Impressively, 


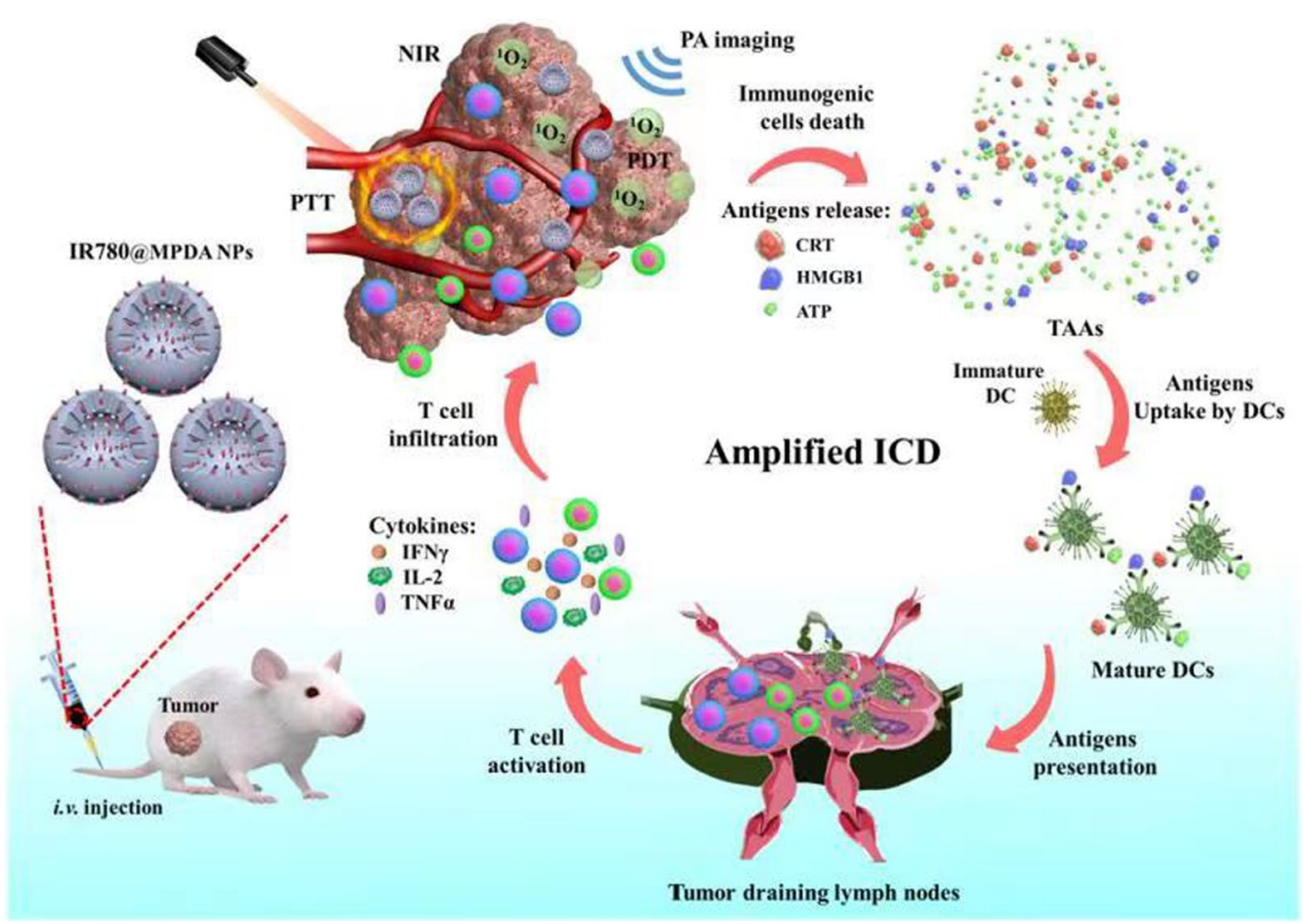

Scheme 1 Illustration of PA imaging-guided synergistic phototherapy and immunotherapy triggered by IR-780@MPDA

an intravenous injection of IR-780@MPDA displayed remarkable tumor growth suppression in 4T1 tumor bearing mice, which is attributed to the combined PDT/ PTT along with the elicitation of immune response, triggering enhanced ICD and CTLs activation (Scheme 1).

\section{Material and methods Materials}

Dopamine hydrochloride, ammonia aqueous solution $\left(\mathrm{NH}_{4} \mathrm{OH}, 28-30 \mathrm{wt} \%\right)$, ethanol, pluronic F127, 1, 3, 5-trimethylbenzene, and IR-780 were purchased from Sigma-Aldrich (Shanghai, China). Mouse breast cancer cells (4T1) were purchased from American Type Culture Collection (ATCC, Manassas, VA). CD3, CD4, CD8 flow cytometric antibodies and their homotypic antibodies were obtained from BD Biosciences (Shanghai, China). Anti-calreticulin (CRT) antibody was obtained from Abcam (Shanghai, China). The enzyme linked immunosorbent assay (ELISA) kits for the detection of adenosine triphosphate (ATP), high-mobility group box 1 (HMGB1), tumor necrosis factor (TNF- $\alpha)$, interferon $\gamma$ (IFN- $\gamma$ ), interleukin-2 (IL-2), and 3-(4, 5-dimethylthiazol-2-yl)-2, 5-diphenyltetrazolium bromide (MTT) were obtained from KeyGEN Biotech. Co., Ltd. (Jiangsu, China). Tumor dissociation kit of mouse was purchased from Miltenyi Biotec (Germany). Fetal bovine serum (FBS), trypsin, Roswell Park Memorial Institute 1640
(RPMI 1640) culture medium, and phosphate buffer saline (PBS) were purchased from Gibco/Life Technologies (Shanghai, China). Singlet oxygen sensor green (SOSG) kits were purchased from Invitrogen (Shanghai, China).

\section{Synthesis of MPDA}

Dopamine hydrochloride $(0.5 \mathrm{~g})$ and F127 (1.0 g) were added into $100 \mathrm{~mL}$ of mixture (water:ethanol $=1: 1$ ), and stirred until the powder was well-dissolved. Then, $2.0 \mathrm{~mL}$ of trimethylbenzene was slowly injected into the mixture at $500 \mathrm{rpm}$ for $30 \mathrm{~min}$ at room temperature. When white turbid liquid was formed, $5.0 \mathrm{~mL}$ of $\mathrm{NH}_{4} \mathrm{OH}$ was added drop by drop into the above mixture to initiate the selfpolymerization of dopamine polymer nanosphere. After continuous reaction, the formed MPDA were centrifuged at 14,000 rpm for $20 \mathrm{~min}$ and washed for 3 times by centrifugation [34].

\section{Loading capacity of IR-780}

For IR-780@MPDA, IR-780 was added into the MPDA solutions and mixed in the dark for $12-24 \mathrm{~h}$. To determine the loading capacity of IR-780, $1 \mathrm{~mL}$ of MPDA $(100 \mu \mathrm{g} /$ $\mathrm{mL}$ ) was dissolved in DI water and mixed with different weight of IR-780 $(100,50,25,12.5,6.25 \mu \mathrm{g})$, respectively. The mixture with different weight ratios was stirred continuously for $24 \mathrm{~h}$. Then, all the product solutions were 
purified by centrifugation at $14,000 \mathrm{rpm}$ for $20 \mathrm{~min}$. After stirring and washing, unconjugated IR-780 was collected and quantified according to the standard curve. The final loading capacity was calculated by the following equation: (weight of total IR-780 - weight of free IR-780)/ (weight of total IR-780 - weight of free IR-780 + weight of MPDA).

\section{Characterization}

Spherical morphology and the size of MPDA and IR-780@MPDA were observed by transmission electron microscopy (TEM, JEOL JEM-2100, Japan). The absorption spectra of IR-780, MPDA, and IR-780@MPDA were recorded using an ultraviolet-visible (UV-Vis) spectrophotometer (Lambda 35, PerkinElmer, USA). The hydrodynamic diameter and the surface charge of IR-780, MPDA, and IR-780@MPDA were determined using a Zeta PALS analyzer (Brookhaven Instruments Co., Holtsville, NY, USA). The fluorescence intensity of IR-780 and IR-780@MPDA was observed using an IVIS Lumina XR system (Caliper, Alameda, CA, USA). The fluorescence spectra of IR-780 and IR-780@MPDA were further obtained by fluorescence spectrophotometer (F-4600, HITACHI, Japan). Nitrogen sorption isotherms of the MPDA were carried out, and the surface area was calculated by Brunauer-Emmett-Teller (BET) method. The pore size was calculated using Barrett-Joyner-Halenda (BJH) method. IR-780@MPDA was dispersed in different physiological solutions $\left(\mathrm{H}_{2} \mathrm{O}\right.$, saline, RPMI 1640, and FBS) for $24 \mathrm{~h}$, and the UV spectroscopy and DLS analysis were performed to estimate the solubility and stability.

\section{Photodynamic and photothermal properties}

To test the PTT properties, $1 \mathrm{~mL}$ IR-780, MPDA, and IR-780@MPDA with equal proportion concentrations were irradiated with an $808 \mathrm{~nm}$ wavelength laser at $1 \mathrm{~W} / \mathrm{cm}^{2}$ for $300 \mathrm{~s}$ (Hi-Tech Optoelectronics Co., Ltd., China). Deionized water $(1 \mathrm{~mL})$ was used as a control. Similarly, laser power $(0,0.2,0.5,1$, and $2 \mathrm{~W} /$ $\left.\mathrm{cm}^{2}\right)$ and concentration $(12.5,25,50,100$, and $200 \mu \mathrm{g} /$ $\mathrm{mL}$ ) dependent photothermal effect were also recorded under $808 \mathrm{~nm}$ laser irradiation for $300 \mathrm{~s}$. To further investigate the photostability, $1 \mathrm{~mL}$ of $100 \mu \mathrm{g} / \mathrm{mL}$ IR-780@MPDA or $40 \mu \mathrm{g} / \mathrm{mL}$ IR-780 was exposed to the NIR laser at a power density of $1 \mathrm{~W} / \mathrm{cm}^{2}$ for five laser ON-OFF cycles (ON, $300 \mathrm{~s}$; OFF, 480 s). The corresponding temperature changes during laser irradiation were monitored in real time using an infrared camera (MAGNITY f15F1, Wuhan VST Light \& Technology Co., Ltd., China). To determine the photodynamic properties, the generation of singlet oxygen by IR-780, MPDA, and IR-780@MPDA was determined from the fluorescence signal of SOSG (excitation: $504 \mathrm{~nm}$, emission: $525 \mathrm{~nm}$ ) after irradiation of NIR laser for 1, 3, and 5 min.

Then the singlet oxygen quantum yield of IR-780@ MPDA in deionized water was measured using indocyanine green (ICG) as a standard. The same proportional concentration solutions of IR-780@MPDA or ICG were mixed with SOSG $(6 \mu \mathrm{g} / \mathrm{mL})$, respectively. The mixture were prepared and exposed to $808 \mathrm{~nm}$ laser irradiation for $0,15,30$, and $60 \mathrm{~s}$. The UV absorption of ICG (at $780 \mathrm{~nm}$ ) and the fluorescence of SOSG were measured after each irradiation. Finally, the singlet oxygen quantum yield of IR-780@MPDA was calculated using the following formula:

$$
\begin{aligned}
\Phi_{I R-780 @ M P D A}= & \Phi_{I C G} K_{I R-780 @ M P D A} A_{I C G} / \\
& K_{I C G} A_{I R-780 @ M P D A}
\end{aligned}
$$

( $\Phi_{I C G}$ : the singlet quantum yield of ICG, $K_{I R-780}$ : the rate constant of SOSG in the presence of IR-780@MPDA, $K_{I C G}$ the rate constant of SOSG in the presence of ICG, $A_{I R-780 @ M P D A}$ : the UV absorption of IR-780@MPDA, $A_{I C G}$ : the UV absorption of ICG).

\section{Assessment of photothermal conversion efficiency}

Aqueous suspensions of MPDA $(100 \mu \mathrm{g} / \mathrm{mL}, 1 \mu \mathrm{mL})$, IR-780@MPDA (100 $\mu \mathrm{g} / \mathrm{mL}, 1 \mathrm{~mL})$, and IR-780 (40 $\mu \mathrm{g} /$ $\mathrm{mL}, 1 \mathrm{~mL})$ were irradiated by a NIR laser $\left(1 \mathrm{~W} / \mathrm{cm}^{2}\right)$ for $5 \mathrm{~min}$, and then cooled down naturally. The photothermal conversion efficiency (PTCE) was calculated using the following equation:

$$
\eta=\frac{h S\left(T_{\max }-T_{a m b}\right)-Q_{0}}{I\left(1-10^{-A}\right)} \times 100 \%
$$

( $h$ : heat transfer coefficient, $S$ : the exposed surface area of a cuvette, $T_{\max }$ : maximum temperature at equilibrium, $T_{\text {amb }}$ : minimum temperature at equilibrium, $Q_{0}$ : heat absorbed by the container, $I$ : incident laser power in W, $A$ : absorbance at $780 \mathrm{~nm}$ ).

When the energy input is equal to the energy dissipated:

$$
h S=\frac{\sum m_{i} C_{p, i}}{\tau_{s}} \approx \frac{m_{\mathrm{H}_{2} \mathrm{O}} C_{\mathrm{H}_{2} \mathrm{O}}}{\tau_{s}}
$$

( $\tau_{s}$ : the time constant, $m_{\mathrm{H}_{2} \mathrm{O}}$ : the weight of $\mathrm{H}_{2} \mathrm{O}, \mathrm{C}_{\mathrm{H}_{2} \mathrm{O}}$ : the specific heat capacity of $\mathrm{H}_{2} \mathrm{O}$ ).

When the laser is off, the mixture is cooled down:

$$
\mathrm{t}=-\tau_{s} \ln \theta=-\tau_{s} \ln \frac{T-T_{a m b}}{T_{\max }-T_{a m b}}
$$


( $\mathrm{t}$ : the time during the cooling stage, $T$ : the temperature of mixture at this time).

\section{Cell culture and cellular uptake}

According to the ATCC's protocol, 4T1 cells were cultured in RPMI 1640 under a $5 \% \mathrm{CO}_{2}$ atmosphere at $37^{\circ} \mathrm{C}$. When the $4 \mathrm{~T} 1$ cell concentration reached $70 \%$, cells were incubated with IR-780@MPDA (100 $\mu \mathrm{g} / \mathrm{mL})$ for 8, 12, and $24 \mathrm{~h}$, while, untreated cells were taken as a control. After cells washing and collection, the fluorescence signals from IR-780 were recorded using a flow cytometer (CytoFLEX, Beckman Coulter, USA) and analyzed by FlowJo v10 software (FlowJo LLC, Ashland, OR, USA).

\section{Detection of cellular ROS}

For intracellular ROS detection, 4T1 cells were cultured in six-well plates. When the cells concentrations reached 70-80\%, cells were incubated with IR-780, MPDA, and IR-780@MPDA with an equal proportion concentrations for $24 \mathrm{~h}$, and untreated cells were taken as a control. After incubation, the cells in treatment groups were collected to further irradiate with an $808 \mathrm{~nm}$ wavelength laser at $1.0 \mathrm{~W} / \mathrm{cm}^{2}$ for $300 \mathrm{~s}$. All cells were washed, collected, and then incubated with ROS fluorescence probe for 30 min according to the manufacturer's protocol. The fluorescence signal was recorded by flow cytometer.

\section{Cell viability assay}

For cellular viability, 4T1 cells were seeded in 96-well plates, and incubated with IR-780, MPDA or IR-780@ MPDA using the same concentration gradient $(0,25,50$, 100 , and $200 \mu \mathrm{g} / \mathrm{mL}$ ) for $24 \mathrm{~h}$ with/without irradiation (808 nm laser, $1.0 \mathrm{~W} / \mathrm{cm}^{2}, 300 \mathrm{~s}$ ). After washing for three times, the cellular viability was measured by MTT assay.

\section{Western blot analysis}

As the ICD process is accompanied by the release of CRT, western blot analysis was performed to observe the expression of CRT. Cells after various treatments were collected for protein quantitative analysis and protein denaturation. Next, the cellular proteins were separated by electrophoresis and electroblotted to hydrophobic polyvinylidene difluoride (PVDF) membranes. The membranes were then incubated with primary antibodies (anti-CRT, 1:10,000) and secondary antibody. Finally, immunoreactive bands were visualized and quantified using ImageJ software after diaminobenzidine (DAB) coloration. To further evaluate the treatment effects in vivo, western blot analysis was also performed as described above to determine CRT expression in the tumors of each group.

\section{Biodistribution in vivo}

All procedures involving animals were approved by the Institutional Animal Care and Use Committee of Jinling hospital (Nanjing, China). Approximately $5 \times 10^{6} 4 \mathrm{~T} 1$ cells were subcutaneously injected into the right flank of mice to establish animal model. When the tumor sizes reached about $100 \mathrm{~mm}^{3}$, a total of 15 mice were used for in vivo imaging, and were divided into three groups $(\mathrm{n}=5)$. Then, mice were injected intravenously with $100 \mu \mathrm{L}$ of MPDA (1 mg/mL), IR-780@MPDA $(1 \mathrm{mg} / \mathrm{mL})$ or $0.4 \mathrm{mg} / \mathrm{mL}$ of IR-780, respectively. The in vivo biodistribution was examined after intravenous injection for $0,2,4,6,8$, and $12 \mathrm{~h}$ using the IVIS Lumina XR system (excitation: $780 \mathrm{~nm}$, emission: $831 \mathrm{~nm}$ ) and Vevo LAZR Imaging System (Fujifilm VisualSonics, Toronto, Canada, excitation: $780 \mathrm{~nm}$ ), respectively. The fluorescence or PA signals of tumors were captured and quantified using Living Image Software.

\section{Photothermal effect in vivo}

Tumor-bearing mice were divided into four groups $(\mathrm{n}=5)$, and injected intravenously with $100 \mu \mathrm{L}$ PBS, IR-780 $(0.4 \mathrm{mg} / \mathrm{mL})$, MPDA $(1 \mathrm{mg} / \mathrm{mL})$ or IR-780@ MPDA $(1 \mathrm{mg} / \mathrm{mL})$ as described above. After the NIR fluorescence and PA imaging guidance, mice were anesthetized and then exposed to an $808 \mathrm{~nm}$ wavelength laser at a power density of $1 \mathrm{~W} / \mathrm{cm}^{2}$ for $300 \mathrm{~s}$. The temperature changes in the tumors were monitored using a MAGNITY f15F1 infrared camera.

\section{Photodynamic/photothermal therapy in vivo}

Tumor-bearing mice were divided into four groups $(\mathrm{n}=10)$. Mice received an intravenous injection of $100 \mu \mathrm{L}$ PBS, IR-780 (0.4 mg/mL), MPDA (1 mg/mL) or IR-780@ MPDA $(1 \mathrm{mg} / \mathrm{mL})$, respectively. Then, under NIR fluorescence and PA imaging guidance, mice were irradiated by NIR laser $\left(808 \mathrm{~nm}, 1 \mathrm{~W} / \mathrm{cm}^{2}\right.$, and $\left.300 \mathrm{~s}\right)$ after $6 \mathrm{~h}$ postintravenous injection.

\section{Blood indicators}

Tumor-bearing mice were divided into four groups $(\mathrm{n}=3)$. After intravenous injection with $100 \mu \mathrm{L}$ PBS, IR-780, MPDA or IR-780@MPDA for $48 \mathrm{~h}$ as described above. The whole blood of each mouse was drawn to test the important biochemical indicators, such as aspartate aminotransferase (AST), alanine aminotransferase (ALT), alkaline phosphatase (ALP), blood urea nitrogen (BUN), and creatinine (CRE).

\section{Histopathology}

After in vivo tumor therapy, tumor, heart, liver, spleen, lungs, and kidneys from each group were dissected and 
prepared for pathological sectioning. All the section specimens were stained with hematoxylin and eosin $(H \& E)$ staining. Besides, tumor specimens were further subjected to vascular immunohistochemical staining (IHC).

\section{Cytotoxic T lymphocytes (CTLs) responses}

After tumor therapy, tumors were collected from all mice. Then, the total lymphocytes were separated from the mice tumors according to the manufacturer's instructions. About $10^{6}$ lymphocytes in each sample were chosen to incubate with CD3, CD4, CD8 antibodies and their homotypic antibodies in the dark for $30 \mathrm{~min}$. The percentage number of cytotoxic $\mathrm{T}$ lymphocytes in each group was further recorded by flow cytometer.

\section{Enzyme linked immunosorbent assay (ELISA)}

ATP and HMGB1 are the important release markers of ICD. Whereas, cytokines such as TNF- $\alpha$, IFN- $\gamma$ and IL-2, are considered as the crucial markers for CTLs activation-induced systemic immune response. In order to test the marker expression after combined therapy, supernatant after cell treatment or serum samples from the mice of each group were prepared and analyzed by ELISA kits according to the manufacturer's protocols.

\section{Statistical analysis}

Statistical analysis was performed using the two-sided Student's t-test for two groups, and two-way analysis of variance for multiple groups using the $\mathrm{R}$ Programming Language. Probabilities as $p<0.05\left(^{*}\right)$ and $p<0.01\left(^{* * *}\right)$ are marked in relevant figures.

\section{Results and discussion}

\section{Preparation and characterization}

Transmission electron microscopic (TEM) characterization revealed spherical shape MPDA with an average diameter of $60 \mathrm{~nm}$ (Fig. 1A). After IR-780 loading, the size and dispersion of IR-780@MPDA did not change as revealed by TEM (Fig. 1B). The pellet of IR-780@
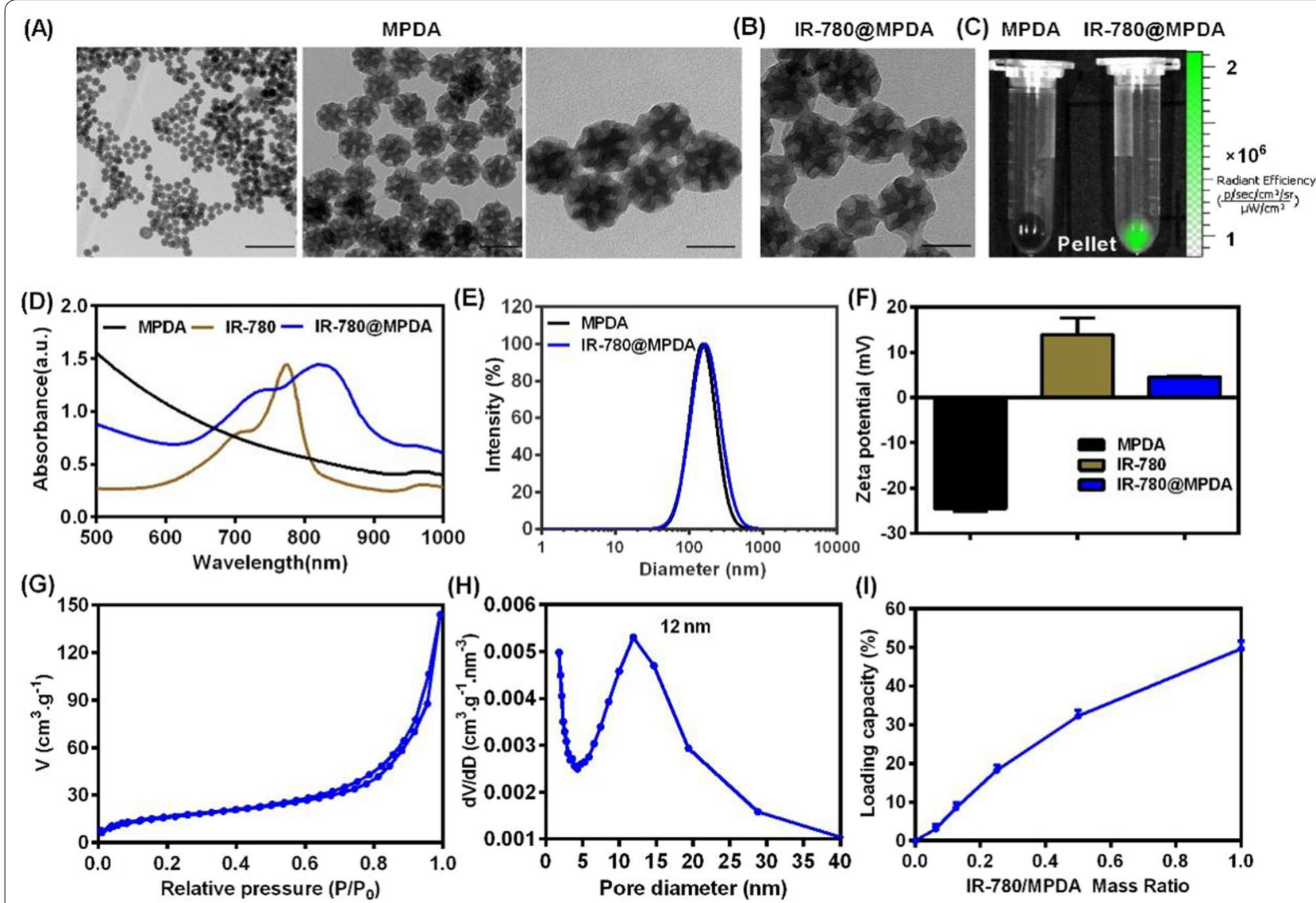

Fig. 1 A TEM images of MPDA; scale bar 500 nm (left), 100 nm (middle), and 50 nm (right). BTEM images of IR-780@MPDA; scale bar 50 nm. CThe pellet of MPDA and IR-780@MPDA after NIR fluorescence imaging. D UV-Vis spectra of MPDA, IR-780, and IR-780@MPDA at the same proportional concentrations. E Hydrodynamic diameter of MPDA and IR-780@MPDA. F The zeta potential of MPDA, IR-780, and IR-780@MPDA. G Nitrogen sorption isotherms of IR-780@MPDA. H Pore size distribution curve of the IR-780@MPDA. I Loading capacity of MPDA 
MPDA after centrifugation exhibited fluorescence signals as shown in Fig. $1 C$, which verified the successful loading of IR-780 onto MPDA. The fluorescence spectra of IR-780 and IR-780@MPDA are presented in Additional file 1: Fig. S2. To further confirm the loading of IR-780, UV-Vis spectra, dynamic light scattering (DLS), and zeta potential measurements were obtained. A characteristic absorption peak of free IR-780 was observed in the absorption spectra of IR-780@MPDA as shown in Fig. 1D, indicating the successful loading of IR-780. On the other hand, after the loading of IR-780, the hydrodynamic diameter of MPDA increased from 152.6 \pm 4.8 to $164.8 \pm 10.2 \mathrm{~nm}$ (Fig. 1E). While, the surface zeta potential shifted from $-24.5 \pm 0.6$ to $4.55 \pm 0.1 \mathrm{mV}$ for IR-780@MPDA, which is attributed to the surface modification of MPDA by positively charged IR-780 (Fig. 1F).

Notably, nitrogen sorption isotherms of the MPDA exhibited a type IV curves in a relative pressure $\left(\mathrm{p} / \mathrm{p}_{0}\right)$ ranging from 0.47 to 0.92 with a large hysteresis loop, suggesting that the MPDA had a typical mesoporous architecture with narrow pore size distribution. The surface area and pore volume were calculated to be as high as $866 \mathrm{~m}^{2} / \mathrm{g}$ and $0.4 \mathrm{~cm}^{3} / \mathrm{g}$, respectively (Fig. $1 \mathrm{G}$ ). The pore size calculation by $\mathrm{BJH}$ method revealed that the MPDA nanoparticles had a uniform mesopores of about $12 \mathrm{~nm}$ (Fig. 1H). To investigate the dye loading capacity of MPDA, different concentrations of IR-780 were mixed with MPDA, and the amount of free IR-780 in the supernatants was quantified. As shown in Fig. 1I, the loading capacity of MPDA is directly proportional to the mass ratio (IR-780: MPDA) as the maximum loading capacity (49.7 wt\%) was obtained at 1:1 mass ratio. These results suggested that MPDA had a high loading capacity for IR-780. In order to detect the stability, IR-780@MPDA were dispersed in $\mathrm{H}_{2} \mathrm{O}$, saline, RPMI 1640 and FBS for $24 \mathrm{~h}$. As shown in Additional file 1: Fig. S3A, no particular aggregation was found even after $24 \mathrm{~h}$ in different physiological media. Similarly, DLS analysis and UV spectroscopy neither show any change in the hydrophilic diameter nor in absorption spectra over different times (Additional file 1: Fig. S3B-F). These results indicated excellent solubility and stability of IR-780@MPDA in the physiological environment.

\section{Photodynamic and photothermal properties}

To investigate the applicability of IR-780@MPDA for simultaneous PDT and PTT, we first tested the photothermal property of IR-780, MPDA, and IR-780@MPDA. As shown in Fig. 2A, water (as a control) did not show any particular temperature increment upon NIR laser irradiation. Whereas, IR-780, MPDA, and IR-780@ MPDA demonstrated notable time-dependent temperature elevation. Specifically, an enhanced photothermal activity was recorded by IR-780@MPDA, which is ascribed to the synergistic photothermal effect of IR-780 and MPDA. Moreover, IR-780@MPDA showed laser power-dependent photothermal effect as the maximum temperature $\left(66^{\circ} \mathrm{C}\right)$ was recorded at $2 \mathrm{~W} / \mathrm{cm}^{2}$ (Fig. 2B). Similarly, concentration-dependent temperature elevation was also noticed as at a concentration of $200 \mu \mathrm{g} /$ $\mathrm{mL}$, the temperature reached $\sim 50{ }^{\circ} \mathrm{C}$ under $808 \mathrm{~nm}$ laser irradiation $\left(1 \mathrm{~W} / \mathrm{cm}^{2}\right)$ as shown in Fig. 2 C. Such a temperature enhancement within $300 \mathrm{~s}$ of NIR laser irradiation was sufficient to trigger localized thermal ablation of tumor cells. Notably, IR-780 showed photothermal degradation under five laser on/off cycles, suggesting photothermal instability and photodegradation (Additional file 1: Fig. S4). Fortunately, the photothermal activity was unchanged even after five consecutive laser on/off cycles, suggesting an excellent photothermal stability of IR-780@MPDA to ensure sustained photothermal tumor cell killing (Fig. 2D). IR-780@MPDA exhibited 54.92\% $\operatorname{PTCE}(\eta)$, which is superior to both IR-780 (24.11\%) and MPDA (42.13\%), respectively (Additional file 1: Fig. S5). Notably, the PTCE of IR-780@MPDA is even higher than reported photothermal nanoagents, such as $\mathrm{TiO}_{2} @ \mathrm{PDA}$ Ce6 (32.12\%) [35], $\mathrm{Au}_{2}$ Pt-PEG-Ce6 (31.5\%) [36], and PDA-hemoglobin-Ce6 (47.09\%) [3].

Along with the photothermal activity, photodynamic properties of IR-780@MPDA were evaluated by determining the ${ }^{1} \mathrm{O}_{2}$ production ability via SOSG assay. Figure $2 \mathrm{E}$ revealed an obvious increase in ${ }^{1} \mathrm{O}_{2}$ generation ability after laser irradiation for 1,3 , and $5 \mathrm{~min}$. The SOSG fluorescence signals of IR-780 and IR-780@MPDA were increased rapidly upon laser irradiation, while no noticeable signal increase was detected in $\mathrm{H}_{2} \mathrm{O}$ or MPDA solution, implying that the ${ }^{1} \mathrm{O}_{2}$ generation capacity is solely because of IR-780 molecules rather than MPDA (Fig. 2F). To determine the singlet oxygen quantum yield of IR-780@MPDA, ICG was used as a standard for quantum yield measurement, which was reported to be 0.2 [37]. We found that the UV absorption of IR-780@MPDA from 500 to $1000 \mathrm{~nm}$ is $1.82\left(A_{I R-780 @ M P D A}\right)$ and 1.83 $\left(A_{\text {ICG }}\right)$ for ICG (Additional file 1: Fig. S6A, B). Besides, the rate constant of SOSG in the presence of IR-780@ MPDA $\left(K_{I R-780 @ M P D A}\right)$ and ICG $\left(K_{I C G}\right)$ was 0.0091 and 0.0057 , respectively (Additional file 1: Fig. S6C, D). Thus, the calculated singlet oxygen quantum yield of IR-780@ $\operatorname{MPDA}\left(\Phi_{I R-780 @ M P D A}\right)$ in water was $\sim 0.32$.

\section{Cellular uptake and cellular PDT/PTT efficacy}

Prior to the evaluation of combined therapy in vitro, the cellular uptake of IR-780@MPDA was investigated. After cells incubation for different times $(8,12$ or $24 \mathrm{~h})$, the fluorescence signal from IR-780 was monitored by flow cytometry. As shown in Fig. 3A, B the fluorescence 
(A)

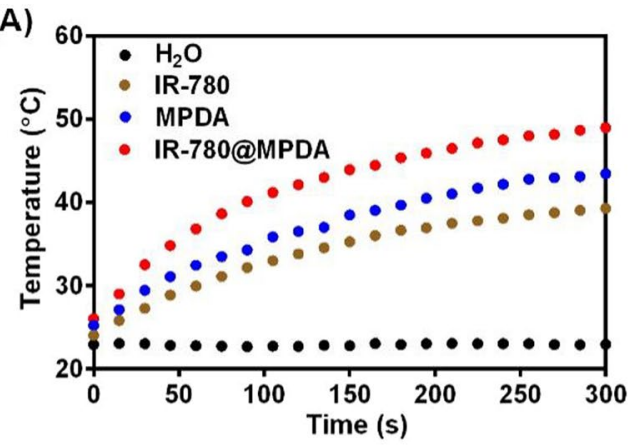

(C)

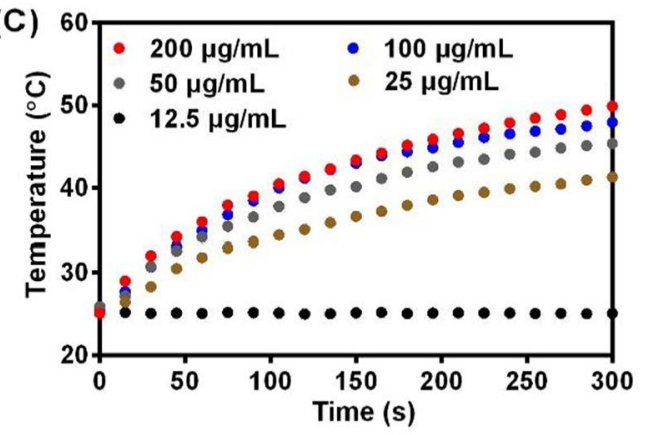

(E)

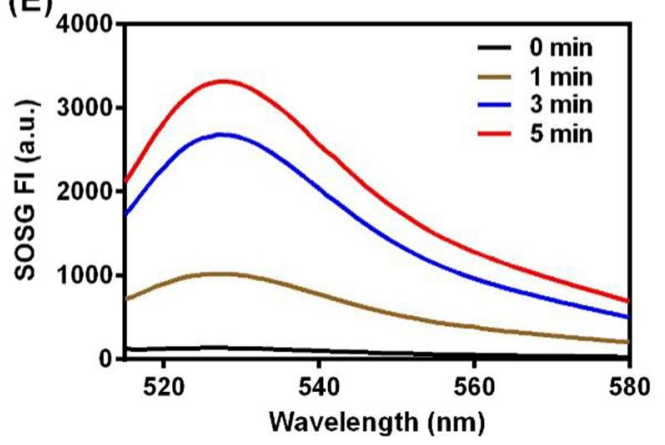

(B)

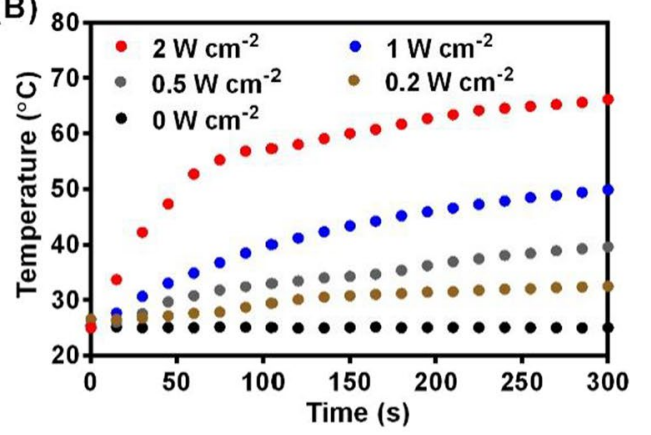

(D)

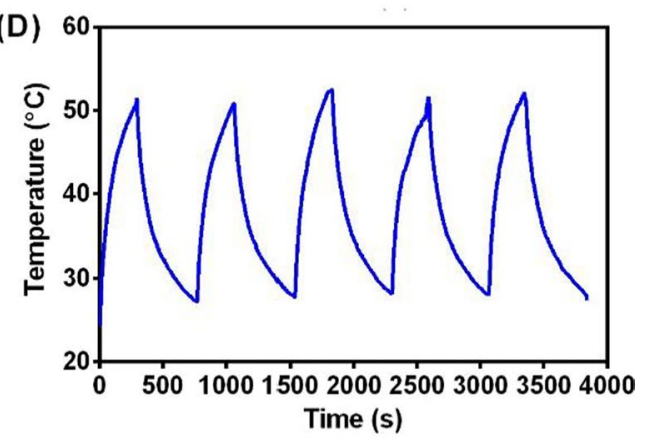

(F)

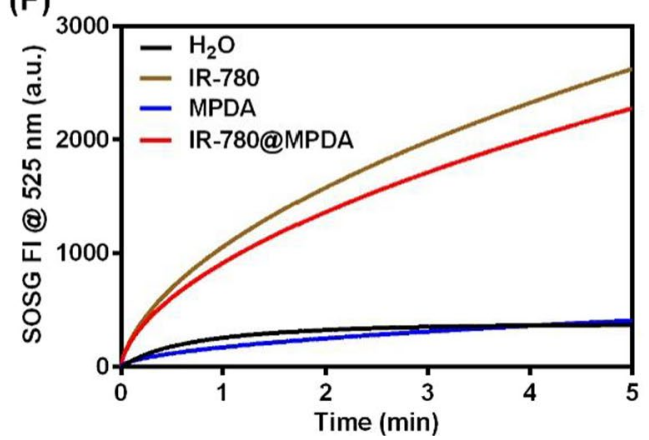

Fig. 2 A Temperature curves of IR-780, MPDA, and IR-780@MPDA at the same proportional concentrations under laser irradiation (808 nm laser, $\left.1 \mathrm{~W} / \mathrm{cm}^{2}, 300 \mathrm{~s}\right) . \mathrm{H}_{2} \mathrm{O}$ was taken as a control. B Temperature curves of IR-780@MPDA at different irradiation powers $\left(0,0.2,0.5,1\right.$, and 2 W/ $\left.\mathrm{cm}^{2}\right) . \mathbf{C}$ Temperature curves of IR-780@MPDA at different concentrations (12.5, 25, 50, 100, and $200 \mathrm{\mu g} / \mathrm{mL}$ ). D Photostability of IR-780@MPDA over five cycles of $808 \mathrm{~nm}$ laser irradiation at $1 \mathrm{~W} / \mathrm{cm}^{2}$ for $300 \mathrm{~s}$ and cooling for $480 \mathrm{~s}$. E SOSG fluorescence intensity (FI) of IR-780@MPDA after different times $(1,3$ and $5 \mathrm{~min})$ of irradiation $\left(808 \mathrm{~nm}, 1 \mathrm{~W} / \mathrm{cm}^{2}\right)$. F SOSG Fl of indicated groups at $525 \mathrm{~nm}$ under NIR laser irradiation

curves are gradually right-shifted upon an increase in the incubation time, and the maximum fluorescence signal is recorded at $24 \mathrm{~h}$, proving the excellent cellular accumulation abilities of IR-780@MPDA, which is favorable for their combined PDT/PTT therapeutic applications.

Intracellular ROS generation was further evaluated in $4 \mathrm{~T} 1$ cells using the $2^{\prime}, 7^{\prime}$-dichlorodihydrofluorescein diacetate (DCFH-DA) probe after incubation with IR-780, MPDA, and IR-780@MPDA in dark or under laser irradiation, respectively. Owing to an excellent intracellular stability, IR-780@MPDA exhibited an enhanced ROS production than free IR-780 or MPDA under NIR irradiation, indicating their high PDT ability at cellular level (Fig. 3C, D). However, without laser excitation, no ROS production was found in each cellular treatment group, suggesting that ROS generation could only be triggered by NIR light activated PDT (Additional file 1: Fig. S7).

The in vitro therapeutic effects against 4T1 tumor cells were further assessed. In dark conditions, more than $80 \%$ cellular viability was observed even at a higher concentration $(200 \mu \mathrm{g} / \mathrm{mL})$ of both MPDA and IR-780@MPDA, implying the good in vitro biocompatibility of MPDA and IR-780@MPDA (Fig. 3E). However, 


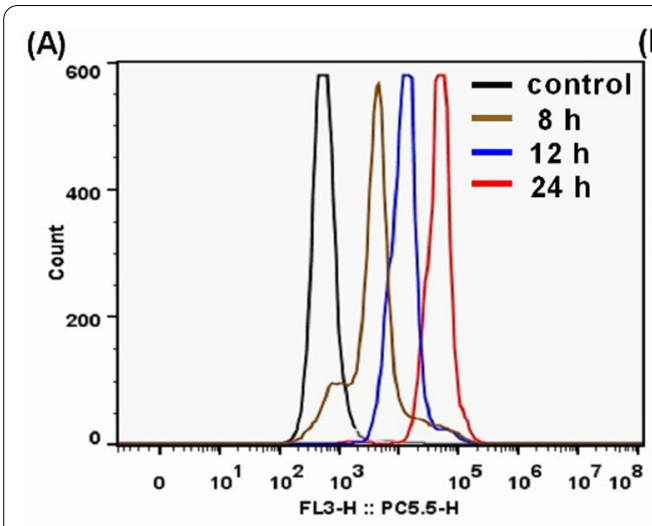

(C)

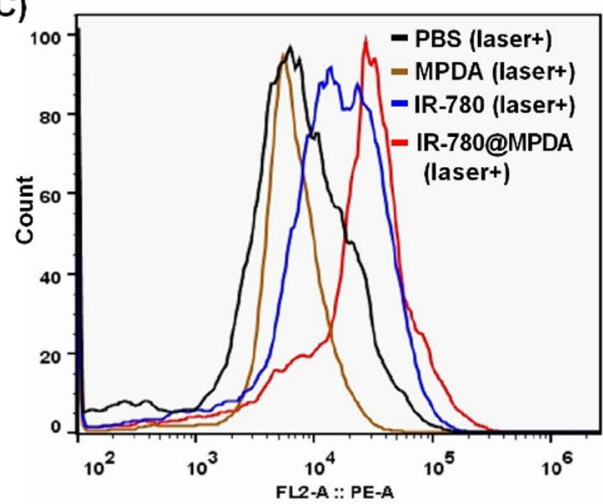

(B)

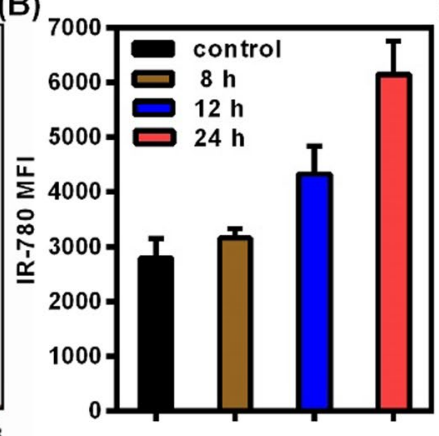

(D)

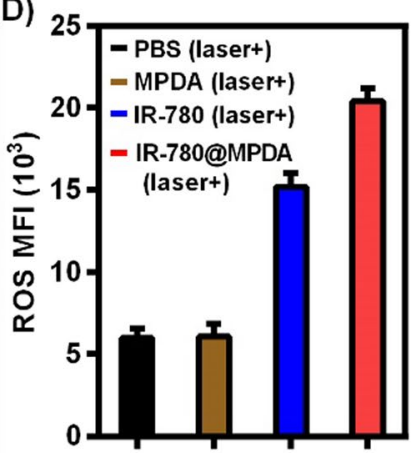

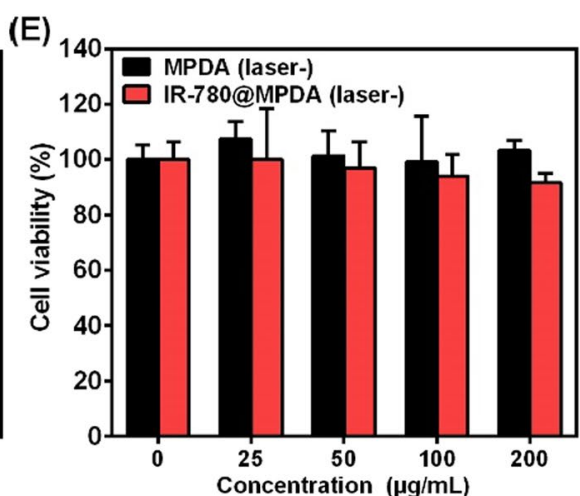

(F)

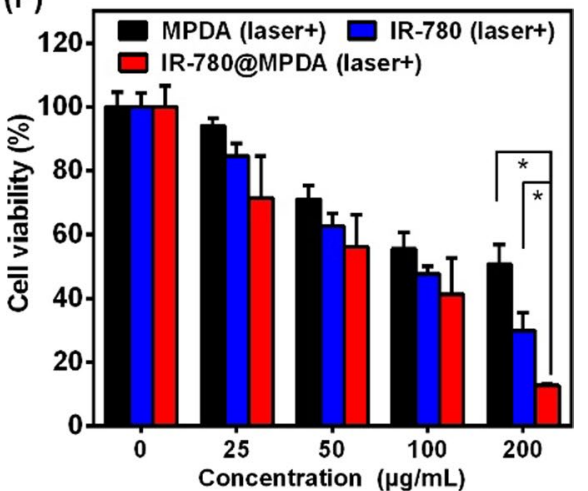

(G)

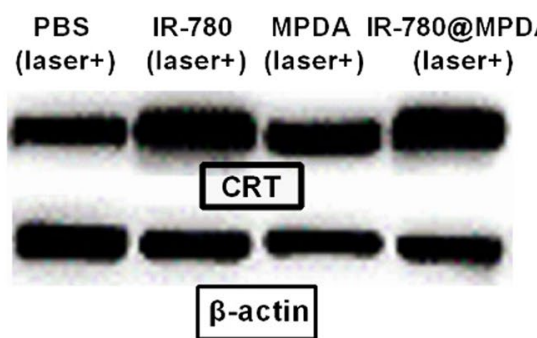

(H)

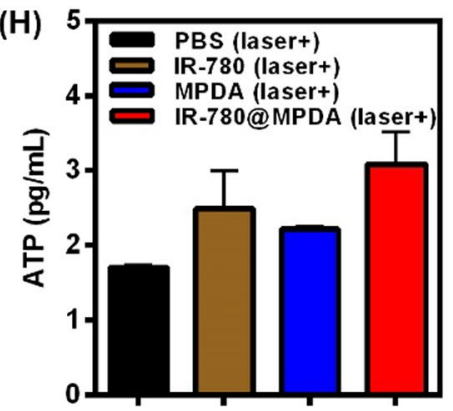

(I)

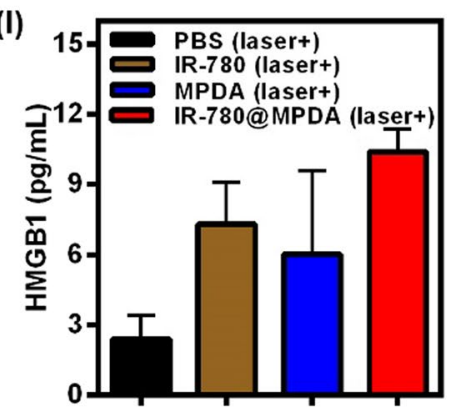

Fig. 3 A The cellular uptake of 4T1 cells after incubation with IR-780@MPDA for 8, 12 or 24 h. B The IR-780 median fluorescence intensity (MFI) at different incubation times. C Intracellular ROS generation in control, MPDA, IR-780, and IR-780@MPDA groups under laser irradiation (808 nm, 1 W/ $\mathrm{cm}^{2}, 300 \mathrm{~s}$ ) as indicated. D The MFI of ROS in each treatment group. E Relative cell viability of 4T1 cells after incubation with different concentrations of MPDA or IR-780@MPDA in dark. F Relative cell viability of 4T1 cells under laser irradiation after incubation with different concentrations of MPDA, IR-780 or IR-780@MPDA as indicated. Asterisk * indicated $p<0.05$. G Western blot analysis of CRT expression in vitro. H Detection of secreted extracellular ATP. I Detection of the released extracellular HMGB1

under $808 \mathrm{~nm}$ laser irradiation for $300 \mathrm{~s}$, a concentration-dependent cellular killing was observed because of the combined PDT and PTT effects (Fig. 3F). The cellular viability of 4T1 cells incubated with IR-780@MPDA was decreased from 71.5 to $12.7 \%$ as the concentration increased from 25 to $200 \mu \mathrm{g} / \mathrm{mL}$. Compared to MPDA and IR-780 alone, IR-780@MPDA exhibited superior therapeutic efficacy especially at a concentration of
$200 \mu \mathrm{g} / \mathrm{mL}$ (MPDA vs IR-780@MPDA: $p=0.04$, IR-780 vs IR-780@MPDA: $p=0.04)$. Such a higher therapeutic killing efficiency by IR-780@MPDA is strongly attributed to the high intracellular delivery of IR-780 and effective combined PDT/PTT therapy in vitro.

The ICD process is usually accompanied by the release of CRT, ATP, and HMGB1 [38, 39]. To investigate whether 
IR-780@MPDA could trigger ICD after the combined therapy, western blot analysis was first performed to assess the CRT exposure. As shown in Fig. 3G and Additional file 1: Fig. S8, after laser excitation, a noticeable difference in CRT release is observed among the bands in each group as indicated. Compared with the mean gray values (MGV) of the PBS group $(67.16 \pm 13.63)$, IR-780@MPDA treatment greatly increased the expression of CRT $(\mathrm{MGV}=103.55 \pm 16.77)$ than free IR-780 $(\mathrm{MGV}=97.72 \pm 17.11)$ or $\mathrm{MPDA}(\mathrm{MGV}=86.24 \pm 12.42)$ treatment due to the combined ICD elicitation from PDT and PTT. Similarly, ATP secretion and HMGB1 release in cell supernatant were further assessed by ELISA. Under laser irradiation, IR-780@MPDA treated cells exhibited significantly enhanced expression of ATP and HMGB1, which was remarkably higher than the cells treated with either IR-780 or MPDA, respectively (Fig. 3H, I). All these results proved that IR-780@MPDA could effectively internalize into the tumor cells and elicit significantly amplified ICD for cellular immunity.

\section{Biodistribution in vivo}

The in vivo biodistribution of therapeutic nanoagent is highly important to ensure targeted tumor accumulation and negligible off-target toxicity in vivo [40]. 4T1 mice model were established to trace the biodistribution of intravenously (i.v.) injected IR-780 or IR-780@ MPDA through an animal optical imaging system. As shown in Fig. 4A, the fluorescence intensities are mainly distributed in the mice abdomen before injection, while, a notable fluorescence signal was seen in the tumor at $2 \mathrm{~h}$ post-injection, which got stronger at $6 \mathrm{~h}$, and then decreased at $8 \mathrm{~h}$, especially in the IR-780@MPDA injected group. Specifically, IR-780@MPDA demonstrated 1.6-fold higher accumulation than mice treated with free IR-780 at $6 \mathrm{~h}$ post-injection ( $p=0.04$, Fig. $4 \mathrm{~B})$, which is possibly because IR-780@MPDA could sustain an effective intratumoral concentration than free IR-780. The enhanced tumor accumulation capability of IR-780@ MPDA was highly favorable for tumor therapy in vivo.

Because MPDA and IR-780 possess photothermal activity, the potential of IR-780@MPDA for noninvasive PA imaging in vivo was further evaluated. As

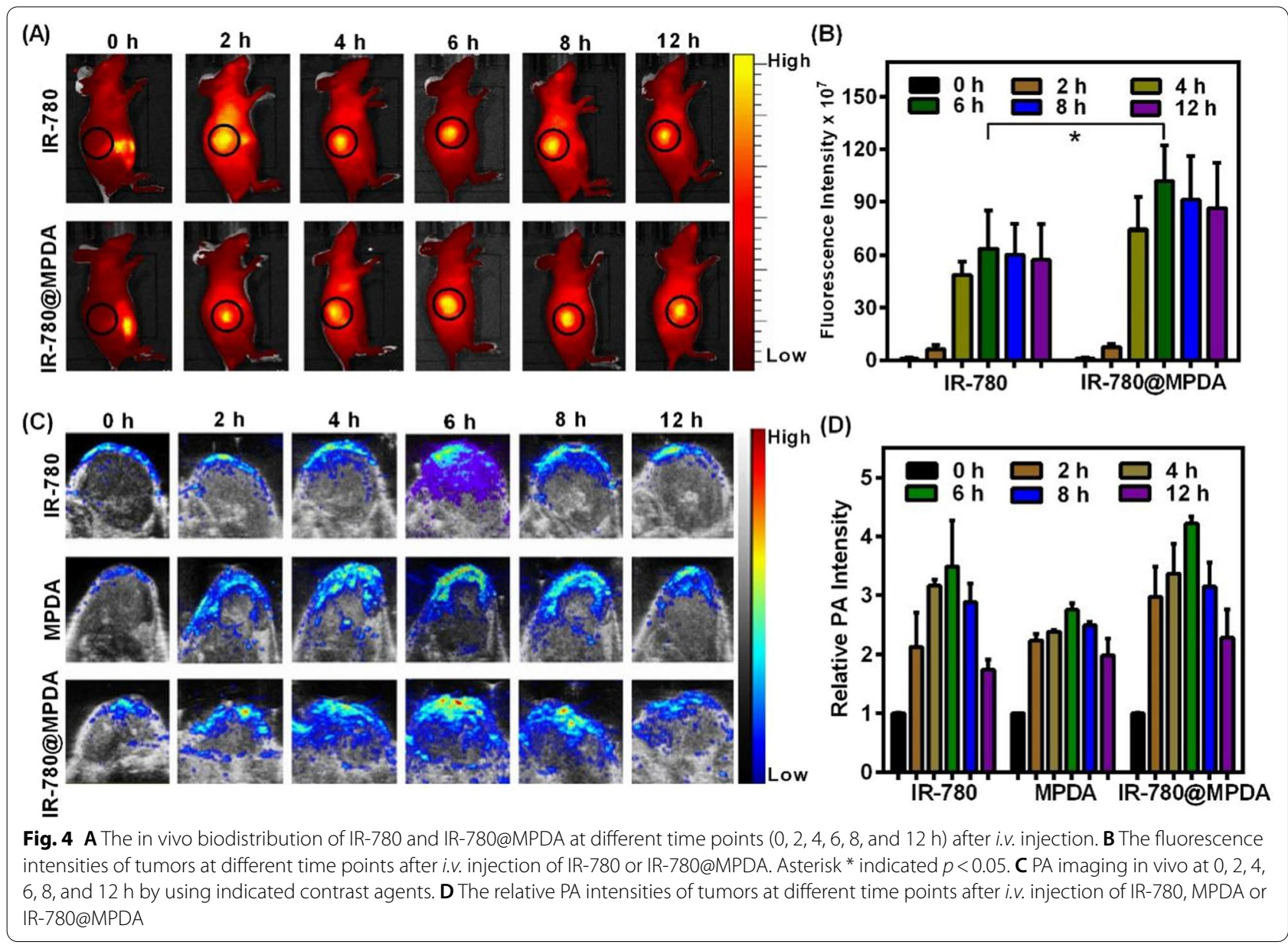


shown in Fig. 4C, D, IR-780, MPDA or IR-780@MPDA displayed time-dependent enhanced PA signals, and the maximum PA signal intensity is recorded at $6 \mathrm{~h}$ post-injection. Due to the EPR effect, IR-780@MPDA exhibited higher tumor accumulation than free IR-780 and MPDA alone, respectively. The in vivo biodistribution traced by PA imaging was in accordance with the NIR fluorescence imaging. Considering the potential advantages of multimodal imaging, the combined treatment could be guided more precisely to achieve desired therapeutic effects at an optimal treatment time.

\section{Combined therapy and ICD in vivo}

To investigate the most effective PTT effect with minimal side effects in vivo, 4T1 tumor-bearing mice were injected intravenously with PBS, IR-780, MPDA or IR-780@MPDA, and then irradiated with $808 \mathrm{~nm}$

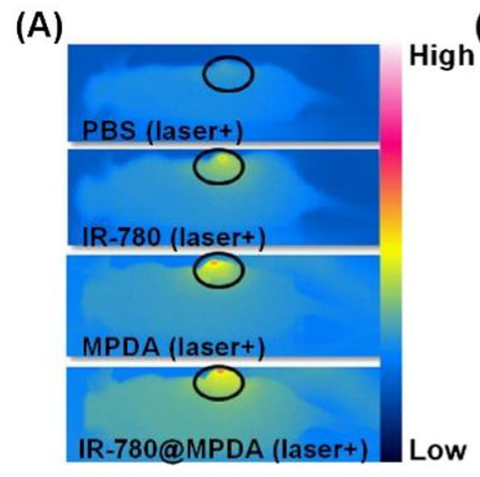

(B)

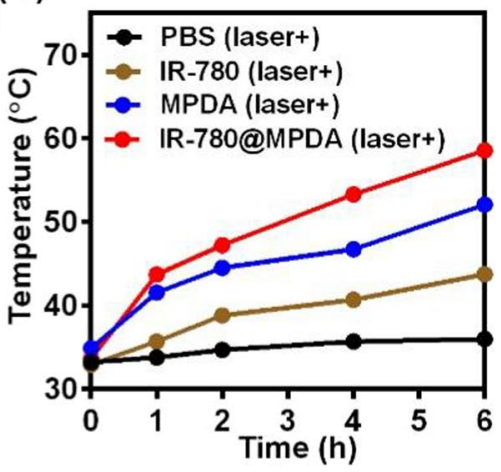

(D)

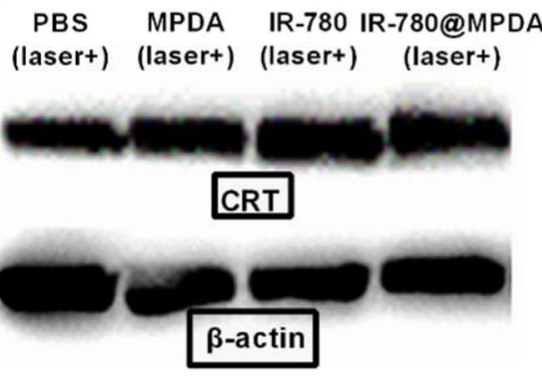

(E)

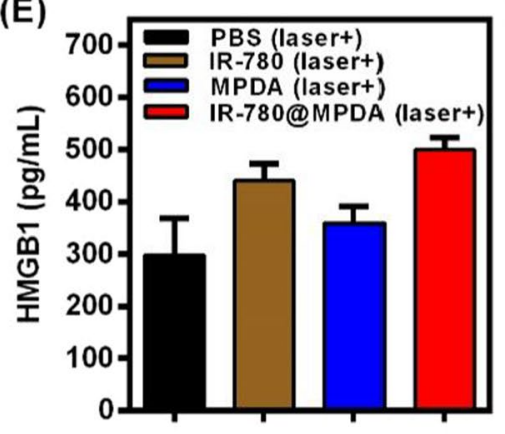

(C)

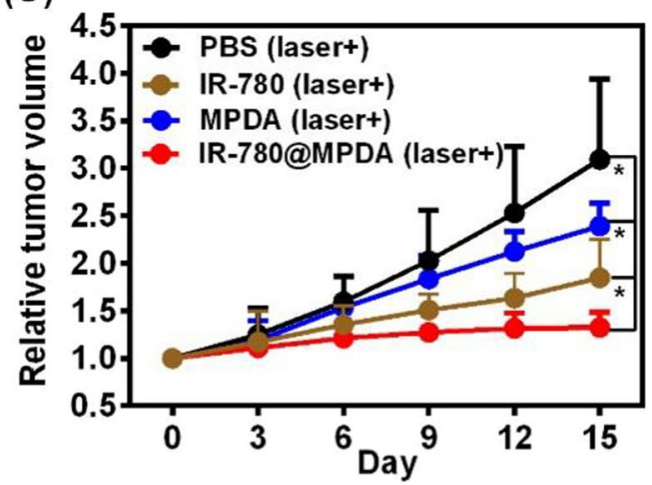

$(\mathrm{F})$

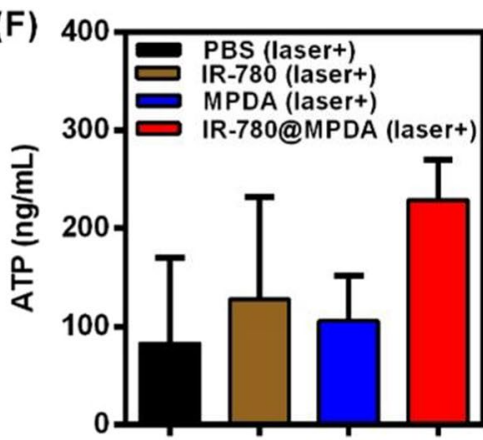

(G)

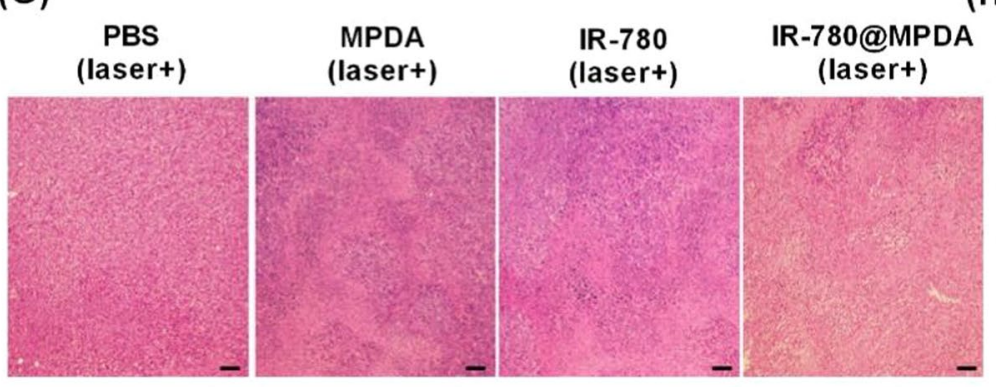

(H)

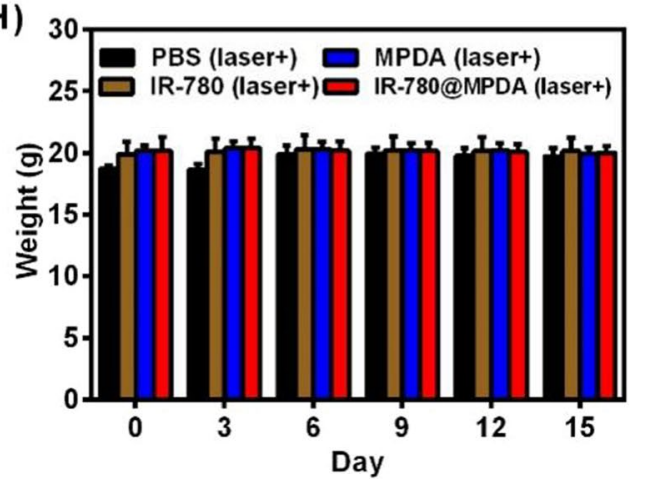

Fig. 5 A The representative thermal images of tumor-bearing mice after i.v. injection of PBS, IR-780, MPDA, and IR-780@MPDA at 6 h. B Tumor temperature elevation under laser irradiation (808 nm, 1 W/ $\mathrm{cm}^{2}$, and $300 \mathrm{~s}$ ) in mice after i.v. injection of PBS, IR-780, MPDA, and IR-780@MPDA at 1, 2,4 , and 6 h. $C$ Relative tumor volume of tumor-bearing mice after indicated treatments. The tumor volumes were normalized to their initial sizes. Asterisk * indicated $p<0.05$. D Western blot analysis of CRT expression of tumor after different treatments. E Detection of HMGB1 release in vivo. $\mathbf{F}$ Detection of ATP secretion in vivo. G H\&E staining of tumors after indicated treatments, scale bars: $100 \mu \mathrm{m}$. H Changes in the body weight of mice after indicated treatments 
laser at a power density of $1 \mathrm{~W} / \mathrm{cm}^{2}$ for $300 \mathrm{~s}$ at 1,2 , 4 , and $6 \mathrm{~h}$ post-injection time points under NIR fluorescence/PA imaging guidance (Fig. 5A, B). Compared to the negligible temperature change in the PBStreated group, a significant temperature increase was seen in the mice injected with other treatment agents. Whereas, the maximum temperature was recorded in the tumor region at $6 \mathrm{~h}$ post-injection because of the maximum tumor enrichment, which was in accordance with the imaging guidance. At $6 \mathrm{~h}$ post-irradiation, the temperature increase was about 15,21 , and $27{ }^{\circ} \mathrm{C}$ in the groups treated with IR-780, MPDA, and IR-780@ MPDA. Notably, due to the tumor-accumulation effect and the low power laser density, there was a minimal injury to the normal skins only around the irradiated tumor areas (Additional file 1: Fig. S9). Hence, IR-780@ MPDA exhibited superior treatment effects due to the synergistic phototherapeutic ability of IR-780 and MPDA, respectively.

The combined PDT/PTT therapeutic efficacy in vivo was further evaluated to verify the antitumor performance of IR-780@MPDA. Tumor volume of 4T1 tumor bearing mice was monitored for 15 days (Fig. 5C). Compared to the control group, mice treated with IR-780@ MPDA displayed significant suppression of tumor growth. The therapeutic efficacy of IR-780@MPDA was remarkably greater than free IR-780 $(p=0.02)$ and MPDA alone $(p=0.02)$.

In order to investigate the combined phototherapy induced ICD in vivo, the crucial ICD biomarkers like CRT, ATP, and HMGB1 were further determined after different treatments for 2 weeks. As expected, the expression level of CRT was remarkably increased by the treatment of IR-780@MPDA (MGV=99.47 \pm 17.11$)$, which was notably higher than free IR-780 $(\mathrm{MGV}=90.10 \pm 11.43)$ or MPDA $(\mathrm{MGV}=68.59 \pm 15.60)$ treatment groups (Fig. 5D and Additional file 1: Fig. S10). It is worthwhile to mention that the in vivo findings are in accordance with the western blot analysis. After collecting mice serum from each treatment group, the release of ATP and HMGB1 were detected by ELISA. Figure $5 \mathrm{E}, \mathrm{F}$ showed the enhanced expression of both ATP and HMGB1 after PDT or PTT alone as compared to the control group, while a significant enhancement was recorded after combined PDT/PTT induced by IR-780@ MPDA. All findings proved that IR-780@MPDA could elicit ICD in vivo.

After treatments, H\&E staining of tumor sections was performed, which further confirmed that IR-780@ MPDA treatment caused more severe necrosis than other treatment agents (Fig. 5G). Moreover, the combined PDT/PTT therapy by IR-780@MPDA inhibited the growth of tumor blood vessels, implying that synergistic phototherapy might influence tumor angiogenesis (Additional file 1: Fig. S11). No apparent weight loss was noticed during the treatment and observation period as shown in Fig. 5H. These findings suggested that mesoporous dopamine delivery nanoplatform exhibited superior synergistic antitumor therapeutic effects.

\section{Immune response in vivo}

Previous studies demonstrated that ICD could generate synergistic immunological effects, resulting in an enhanced recognition of immune cells [41, 42]. To explore the immune stimulatory effects after PDT/PTT induced by IR-780@MPDA, total lymphocytes were extracted from the tumors of each mice at 15th day posttreatment. As shown in Fig. 6A, the frequency of $\mathrm{CD}^{+}$ $\mathrm{T}$ cells in IR-780@MPDA treated mice group (40.6\%) was significantly higher than IR-780 treated group (29.3\%), MPDA treated group (28.3\%), and PBS treated group (8.5\%), verifying that IR-780@MPDA could evoke greater immunostimulatory effects through the tumorassociated antigens release by PDT/PTT elicited ICD. Moreover, CTLs $\left(\mathrm{CD}^{+}{ }^{+} \mathrm{CD} 4^{-} \mathrm{CD}^{+}\right)$are the important subtypes of $\mathrm{T}$ lymphocytes and could kill tumor cells directly via immunotherapy. Therefore, the relative frequency of CTLs in $\mathrm{CD}^{+}{ }^{+} \mathrm{T}$ cells was explored to identify the immune response effects of IR-780@MPDA. As shown in Fig. 6B, IR-780@MPDA triggered significant stimulation of $\mathrm{CD} 8^{+}$CTLs (24.4\%), which was higher than IR-780 (19.3\%), MPDA (17.7\%), and PBS (13.9\%) after treatments. Quantitative analysis presented in Fig. 6C revealed 2.9-fold percentage of CTLs from IR-780@MPDA than PBS treated group $(p=0.001)$, promoting more lymphocytes to attack tumor cells. Moreover, TNF- $\alpha$, IL-2 and INF- $\gamma$ are important indicators of the activation of cellular immunity, and their enhanced expression was found in the group of IR-780@MPDA under laser irradiation, indicating an effective ICD and immune response (Fig. 6D-F) [43]. Taken together, IR-780@MPDA exhibited superior therapeutic effects not only because of the tumor targeting PDT/PTT effects but also by the enhanced immune stimulatory effects.

\section{Biocompatibility and toxicity evaluation in vivo}

To determine the safety of IR-780, MPDA or IR-780@ MPDA in vivo, healthy mice were chosen and the blood was drawn after $6 \mathrm{~h}$ post-i.v. injection of the as-mentioned treatment agents. The blood biochemical indicators of liver and kidney functions, including AST, ALT, ALP, BUN, and CRE, were determined. As presented in Fig. 7A-C, compared with the control group (injected with PBS), no significant differences were found between the groups injected with MPDA or IR-780@ 


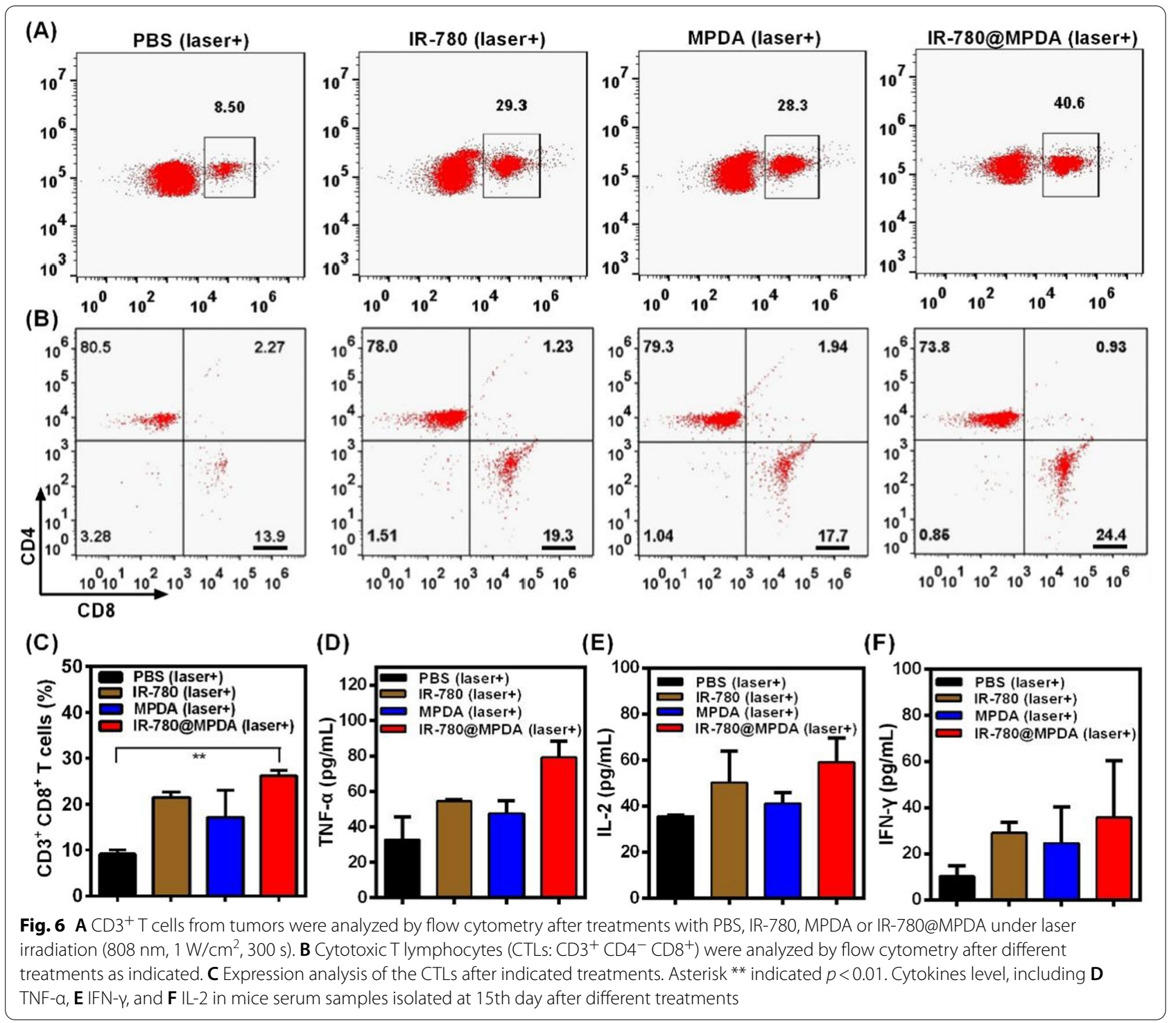

MPDA, further demonstrating the good biosafety in vivo. However, the expressions of AST, ALT, ALP or CRE in mice injected with free IR-780 were higher. The increase of AST, ALT and ALP reflected liver damage and the possible impairment of heart (Fig. 7A), while an increase in CRE level indicated the poor renal function (Fig. 7C). In addition, H\&E staining of the vital organs collected from each group did not reveal an apparent injury except IR-780 group. In IR-780 treated group, the blood vessels of heart, lungs, and liver were expanded with bleeding. Besides, many inflammatory cells were infiltrated in liver and kidneys, while splenic corpuscles were also appeared in spleen (Fig. 7D).
These degeneration and inflammation indicated the potential toxicity of free IR-780, whereas, the integration of IR-780 with MPDA resulted in a significantly reduced toxicity in vivo, and thus IR-780@MPDA with excellent biocompatibility and negligible toxicity are highly suitable for clinical transformation.

\section{Conclusion}

In summary, we established "Two in One" tumor collaborative therapy by mesoporous polydopamine delivery nanoplatform loaded with NIR dye IR-780 (IR-780@ MPDA). IR-780 can serve as a multifunctional agent for duplex NIR fluorescence/PA imaging-guided tumor PDT and PTT in vivo. After loading onto the MPDA, 


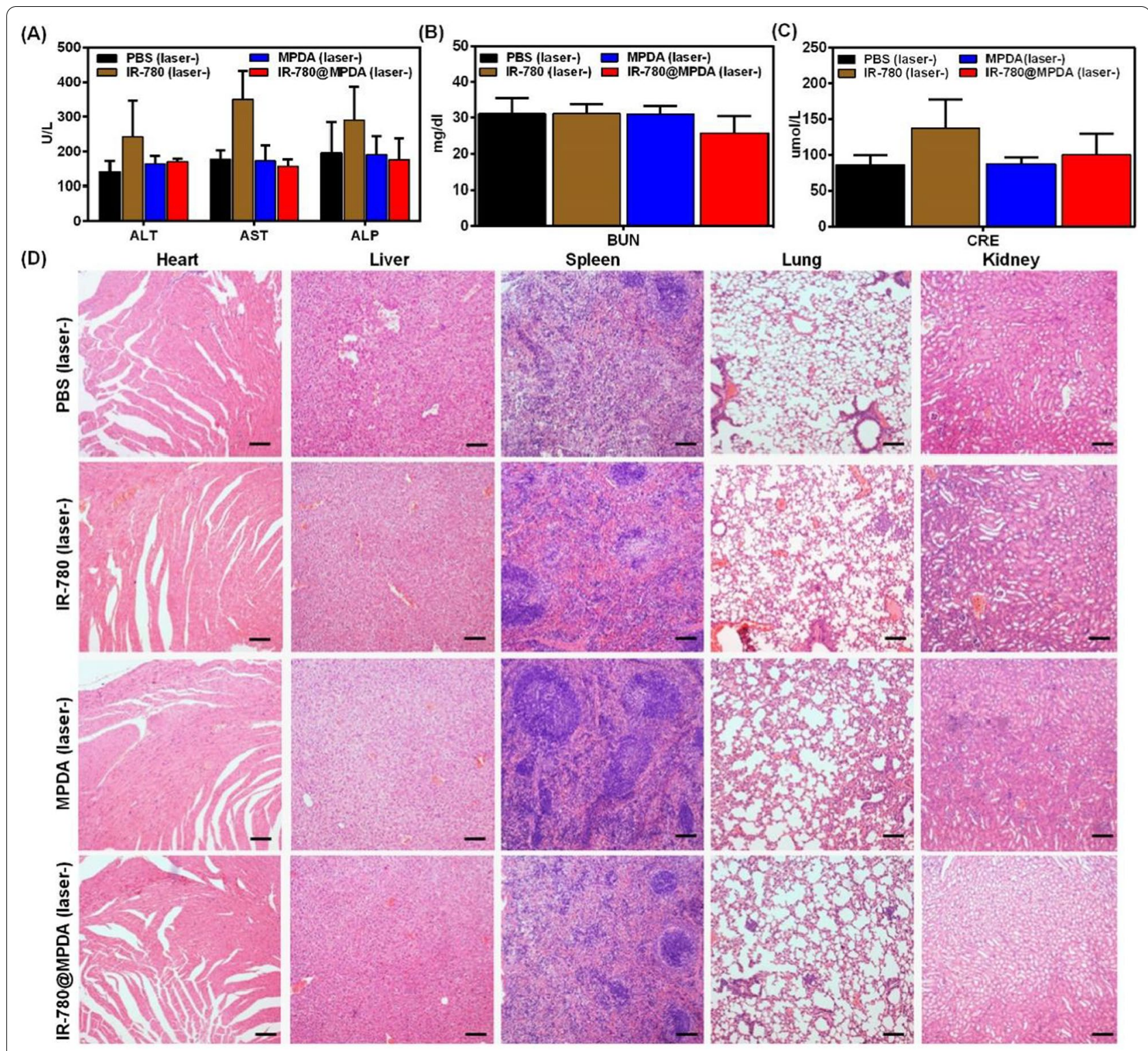

Fig. 7 The blood biochemical analysis after i.v. injection of PBS, IR-780, MPDA or IR-780@MPDA. Data were obtained for A ALT, AST, ALP, B BUN, and C CRE. D H\&E staining of the main organs harvested from mice after $6 \mathrm{~h}$ post-i.v. injection of PBS, IR-780, MPDA or IR-780@MPDA. Scale bars: $100 \mu \mathrm{m}$

the biocompatibility, water-solubility, delivery capacities, and potential toxicity of IR-780 were all greatly improved. Meanwhile, due to the combined effect, IR-780@MPDA triggered synergistic targeted phototherapy, resulting in tumor elimination and microvessels inhibition in vivo. Furthermore, the ICD induced by synergistic PDT/PTT caused the activation of CTLs at tumor sites for potential immunotherapeutic response. Such a compact and practical strategy may further be broadened to other solid tumors to improve the therapeutic efficacy.

\section{Supplementary Information}

The online version contains supplementary material available at https://doi. org/10.1186/s12951-021-01109-7.

Additional file 1: Figure S1. Schematic design of IR-780@MPDA nanoplatform. Figure S2. The fluorescence spectra of IR-780 and IR-780@ MPDA. Figure S3. (A) The digital photographs of IR-780@MPDA dispersions in $\mathrm{H}_{2} \mathrm{O}$, saline, RPMI 1640, and FBS at 0 and $24 \mathrm{~h}$. (B) The hydrodynamic diameters of IR-780@MPDA in various physiological solutions at $0,6,12$, and 24 h. (C-F) UV-Vis spectra of IR-780@MPDA in various physiological solutions as mentioned at 0, 6, 12, and $24 \mathrm{~h}$. Figure S4. Photothermal stability of IR-780 over five laser on/off cycles of $808 \mathrm{~nm}$ laser irradiation at $1 \mathrm{~W} / \mathrm{cm}^{2}$ for $300 \mathrm{~s}$ and cooling for $480 \mathrm{~s}$. Inset is the digital photographs of IR-780 solution before and after NIR laser irradiation for 300 s. Figure S5. (A) Photothermal effect of IR-780@MPDA was recorded 
under NIR laser irradiation for 5 min and then naturally cooled down. (B) Photothermal effect of MPDA. (C) Photothermal effect of free IR-780. (D) Linear time data versus - In $(\theta)$ obtained from the cooling period of (A). (E) Linear time data versus - In $(\theta)$ obtained from the cooling period of (B). (F) Linear time data versus $-\ln (\theta)$ obtained from the cooling period of (C). Figure S6. (A) The absorption spectrum of IR-780@MPDA. (B) The absorption spectrum of ICG. (C) Linear plot of the increased fluorescence intensity ( $525 \mathrm{~nm}$ ) of SOSG in the presence of IR-780@MPDA as the irradiation time. (D) Linear plot of the increased fluorescence intensity ( 525 $\mathrm{nm}$ ) of SOSG in the presence of ICG as the irradiation time. Figure S7. (A) Intracellular ROS generation in control, MPDA, IR-780, and IR-780@MPDA groups under dark conditions. (B) The ROS median fluorescence intensity (MFI) in each group. Figure S8. Mean gray values of CRT bands in each cellular group as indicated. Figure S9. Representative photographs of 4T1 tumor bearing mice treated with PBS, IR-780, MPDA or IR-780@MPDA before and after NIR laser irradiation. The black circles indicated the locations of the tumors. Figure S10. Mean gray values of CRT bands in each treatment group in vivo. Figure S11. Vascular IHC staining of tumors after indicated treatments, scale bars: $100 \mu \mathrm{m}$.

\section{Acknowledgements}

We greatly appreciate the financial support from the National Natural Science Foundation of China (81971681, 81601556, 81530054 and 81901806), National Key R\&D Program of China (2020YFA0908800), Basic Research Program of Shenzhen (JCYJ20180507182413022, JCYJ20200109105620482) and Shenzhen Science and Technology Program (KQTD20190929172538530). We thank Instrumental Analysis Center of Shenzhen University (Lihu Campus).

\section{Authors' contributions}

YTi, MRY and YTa: Investigation, data curation, writing —original draft. XL: data curation. GH: Graphic design. SW and ZT: writing —review and editing. PH, LZ and GL: conceptualization, supervision, funding acquisition, writing — review and editing. All authors read and approved the final manuscript.

\section{Funding}

We greatly appreciate the financial support from the National Natural Science Foundation of China (81971681, 81601556, 81530054 and 81901806), National Key R\&D Program of China (2020YFA0908800), Basic Research Program of Shenzhen (JCYJ20180507182413022, JCYJ20200109105620482) and Shenzhen Science and Technology Program (KQTD20190929172538530).

\section{Availability of data and materials}

All data used to generate these results is available in the main text and additional information.

\section{Declarations}

\section{Ethics approval and consent to participate}

All animal studies were approved by the Institutional Animal Care and Use Committee of Jinling hospital.

\section{Consent for publication}

All authors agree to be published.

\section{Competing interests}

The authors declare no conflict of interests.

\section{Author details}

'Department of Medical Imaging, Jinling Hospital, Medical School of Nanjing University, Nanjing 210002, People's Republic of China. ${ }^{2}$ State Key Laboratory of Analytical Chemistry for Life Science, School of Chemistry and Chemical Engineering, Nanjing University, Nanjing 210093, People's Republic of China. ${ }^{3}$ Marshall Laboratory of Biomedical Engineering, International Cancer Center, Laboratory of Evolutionary Theranostics (LET), School of Biomedical Engineering, Shenzhen University Health Science Center, Shenzhen 518060, People's Republic of China. ${ }^{4}$ Key Laboratory for Organic Electronics and Information Displays \& Institute of Advanced Materials, Nanjing University of Posts \& Telecommunications, Nanjing 210023, People's Republic of China.
Received: 4 August 2021 Accepted: 1 November 2021

Published online: 17 November 2021

\section{References}

1. Jiang BP, Zhang L, Guo XL, Shen XC, Wang Y, Zhu Y, et al. Poly(Nphenylglycine)-based nanoparticles as highly effective and targeted near-infrared photothermal therapy/photodynamic therapeutic agents for malignant melanoma. Small. 2017;13:1602496.

2. Liu B, Li C, Chen G, Liu B, Deng X, Wei Y, et al. Synthesis and optimization of $\mathrm{MoS}_{2} @ \mathrm{Fe}_{3} \mathrm{O}_{4}-\mathrm{ICG} / \mathrm{Pt}(\mathrm{IV})$ nanoflowers for MR/IR/PA bioimaging and combined PTT/PDT/chemotherapy triggered by $808 \mathrm{~nm}$ laser. Adv Sci. 2017:4:1600540.

3. Wang Y, Luo S, Wu Y, Tang P, Liu J, Liu Z, et al. Highly penetrable and on-demand oxygen release with tumor activity composite nanosystem for photothermal/photodynamic synergetic therapy. ACS Nano. 2020;14:17046-52.

4. Zhang Y, Lv F, Cheng Y, Yuan Z, Yang F, Liu C, et al. Pd@Au bimetallic nanoplates decorated mesoporous $\mathrm{MnO}_{2}$ for synergistic nucleus-targeted NIR-II photothermal and hypoxia-relieved photodynamic therapy. Adv Healthc Mater. 2020;9:1901528.

5. Cai Y, Liang PP, Tang QY, Yang XY, Si WL, Huang W, et al. Diketopyrrolopyrrole-triphenylamine organic nanoparticles as multifunctional reagents for photoacoustic imaging-guided photodynamic/photothermal synergistic tumor therapy. ACS Nano. 2017;11:1054-63.

6. Ye SY, Rao JM, Qiu SH, Zhao JL, He H, Yan ZL, et al. Rational design of conjugated photosensitizers with controllable photoconversion for dually cooperative phototherapy. Adv Mater. 2018;30:1801216.

7. Shao W, Yang C, Li FY, Wu JH, Wang N, Ding Q, et al. Molecular design of conjugated small molecule nanoparticles for synergistically enhanced PTT/PDT. Nano-Micro Lett. 2020;12:147.

8. Zhang Q, Wu L, Liu S, Chen Q, Zeng L, Chen X, et al. Targeted nanobody complex enhanced photodynamic therapy for lung cancer by overcoming tumor microenvironment. Cancer Cell Int. 2020;20:570.

9. Chen WH, Luo GF, Zhang XZ. Recent advances in subcellular targeted cancer therapy based on functional materials. Adv Mater. 2019;31:1802725

10. Li W, Yang J, Luo L, Jiang M, Qin B, Yin H, et al. Targeting photodynamic and photothermal therapy to the endoplasmic reticulum enhances immunogenic cancer cell death. Nat Commun. 2019;10:3349.

11. Turubanova VD, Balalaeva IV, Mishchenko TA, Catanzaro E, Alzeibak R, Peskova NN, et al. Immunogenic cell death induced by a new photodynamic therapy based on photosens and photodithazine. J Immunother Cancer. 2019;7:350.

12. Sweeney EE, Cano-Mejia J, Fernandes R. Photothermal therapy generates a thermal window of immunogenic cell death in neuroblastoma. Small. 2018;14:1800678.

13. Ding $B$, Zheng $P$, Jiang $F$, Zhao Y, Wang M, Chang M, et al. MnOx nanospikes as nanoadjuvants and immunogenic cell death drugs with enhanced antitumor immunity and antimetastatic effect. Angew Chem Int Ed. 2020;59:16381-4.

14. Kroemer G, Galluzzi L, Kepp O, Zitvogel L. Immunogenic cell death in cancer therapy. Annu Rev Immunol. 2013;31:51-72.

15. Duan X, Chan C, Lin W. Nanoparticle-mediated immunogenic cell death enables and potentiates cancer immunotherapy. Angew Chem Int Ed. 2019:58:670-80.

16. Jin L, Shen S, Huang Y, Li D, Yang X. Corn-like Au/Ag nanorod-mediated NIR-II photothermal/photodynamic therapy potentiates immune checkpoint antibody efficacy by reprogramming the cold tumor microenvironment. Biomaterials. 2020;268:120582.

17. Zhang X, Tang J, Li C, Lu Y, Cheng L, Liu J. A targeting black phosphorus nanoparticle based immune cells nano-regulator for photodynamic/photothermal and photo-immunotherapy. Bioact Mater. 2021;6:472-89.

18. Zhang C, Liu T, Su Y, Luo S, Zhu Y, Tan X, et al. A near-infrared fluorescent heptamethine indocyanine dye with preferential tumor accumulation for in vivo imaging. Biomaterials. 2010;31:6612-7.

19. Liu M, Zhang P, Deng L, Guo D, Tan M, Huang J, et al. IR780-based lightresponsive nanocomplexes combining phase transition for enhancing multimodal imaging-guided photothermal therapy. Biomater Sci. 2019;7:1132-46. 
20. Zhang Y, He L, Wu J, Wang K, Wang J, Dai W, et al. Switchable PDT for reducing skin photosensitization by a NIR dye inducing self-assembled and photo-disassembled nanoparticles. Biomaterials. 2016;107:23-32.

21. Li M, Li L, Su K, Liu X, Zhang T, Liang Y, et al. Highly effective and noninvasive near-infrared eradication of a staphylococcus aureus biofilm on implants by a photoresponsive coating within $20 \mathrm{~min}$. Adv Sci. 2019;6:1900599.

22. Yang Z, Wang J, Liu S, Li X, Miao L, Yang B, et al. Defeating relapsed and refractory malignancies through a nano-enabled mitochondriamediated respiratory inhibition and damage pathway. Biomaterials. 2020;229:119580

23. Xiao YF, An FF, Chen JX, Yu J, Tao WW, Yu ZQ, et al. The nanoassembly of an intrinsically cytotoxic near-infrared dye for multifunctionally synergistic theranostics. Small. 2019;15:1903121.

24. Yang Z, Wang J, Ai S, Sun J, Mai X, Guan W. Self-generating oxygen enhanced mitochondrion-targeted photodynamic therapy for tumor treatment with hypoxia scavenging. Theranostics. 2019;9:6809-23.

25. Yang ZL, Tian W, Wang Q, Zhao Y, Zhang YL, Tian Y, et al. Oxygen-evolving mesoporous organosilica coated prussian blue nanoplatform for highly efficient photodynamic therapy of tumors. Adv Sci. 2018:5:1700847.

26. Wang J, Sun J, Hu W, Wang Y, Chou T, Zhang B, et al. A porous Au@ Rh bimetallic core-shell nanostructure as an $\mathrm{H}_{2} \mathrm{O}_{2}$-driven oxygenerator to alleviate tumor hypoxia for simultaneous bimodal imaging and enhanced photodynamic therapy. Adv Mater. 2020;32:2001862.

27. Jiang C, Cheng H, Yuan A, Tang X, Wu J, Hu Y. Hydrophobic IR780 encapsulated in biodegradable human serum albumin nanoparticles for photothermal and photodynamic therapy. Acta Biomater. 2015;14:61-9.

28. Deng L, Guo W, Li G, Hu Y, Zhang LM. Hydrophobic IR780 loaded sericin nanomicelles for phototherapy with enhanced antitumor efficiency. Int J Pharm. 2019;566:549-56.

29. Nagy-Simon T, Potara M, Craciun AM, Licarete E, Astilean S. IR780-dye loaded gold nanoparticles as new near infrared activatable nanotheranostic agents for simultaneous photodynamic and photothermal therapy and intracellular tracking by surface enhanced resonant Raman scattering imaging. J Colloid Interface Sci. 2018;517:239-50.

30. Zhou X, Liang J, Liu Q, Huang D, Xu J, Gu H, et al. Codelivery of epigallocatechin-3-gallate and diallyl trisulfide by near-infrared light-responsive mesoporous polydopamine nanoparticles for enhanced antitumor efficacy. Int J Pharm. 2020;592:120020.

31. Li T, Ding B, Wang J, Qin Z, Fernando JFS, Bando Y, et al. Sandwichstructured ordered mesoporous polydopamine/MXene hybrids as high-performance anodes for lithium-ion batteries. ACS Appl Mater Inter. 2020;12:14993-5001.

32. Wang D, Wu H, Zhou J, Xu P, Wang C, Shi R, et al. In situ one-pot synthesis of MOF-polydopamine hybrid nanogels with enhanced photothermal effect for targeted cancer therapy. Adv Sci. 2018;5:1800287.
33. Tian Y, Wang X, Zhao S, Liao X, Younis MR, Wang S, et al. JQ1-loaded polydopamine nanoplatform inhibits C-MYC/programmed cell death ligand 1 to enhance photothermal therapy for triple-negative breast cancer. ACS Appl Mater Inter. 2019;11:46626-36.

34. Peng L, Hung CT, Wang S, Zhang X, Zhu X, Zhao Z, et al. Versatile nanoemulsion assembly approach to synthesize functional mesoporous carbon nanospheres with tunable pore sizes and architectures. J Am Chem Soc. 2019;141:7073-80.

35. Gao Y, Zhang LY, Liu YH, Sun SJ, Yin ZB, Zhang LL, et al. Ce6/Mn ${ }^{2+}$-chelated polydopamine@black-TiO ${ }_{2}$ nanoprobes for enhanced synergistic phototherapy and magnetic resonance imaging in $4 \mathrm{~T} 1$ breast cancer. Nanoscale. 2020;12:1801-10.

36. Wang M, Chang MY, Chen Q, Wang DM, Li CX, Hou ZY, Lin J, et al. $\mathrm{Au}_{2}$ Pt-PEG-Ce6 nanoformulation with dual nanozyme activities for synergistic chemodynamic therapy/phototherapy. Biomaterials. 2020;252:120093.

37. Chen SJ, Huang BY, Pei WJ, Wang L, Xu Y, Niu CC. Mitochondria-targeting oxygen-sufficient perfluorocarbon nanoparticles for imaging-guided tumor phototherapy. Int J Nanomed. 2020;15:8641-58.

38. Krysko DV, Garg AD, Kaczmarek A, Krysko O, Agostinis P, Vandenabeele P. Immunogenic cell death and DAMPs in cancer therapy. Nat Rev Cancer. 2012;12:860-75.

39. Fucikova J, Kasikova L, Truxova I, Laco J, Skapa P, Ryska A, et al. Relevance of the chaperone-like protein calreticulin for the biological behavior and clinical outcome of cancer. Immunol Lett. 2018;193:25-34.

40. Badrigilan S, Heydarpanahi F, Choupani J, Jaymand M, Samadian H, Hoseini-Ghahfarokhi M, et al. A review on the biodistribution, pharmacokinetics and toxicity of bismuth-based nanomaterials. Int J Nanomed. 2020;15:7079-96.

41. Liu H, Hu Y, Sun Y, Wan C, Zhang Z, Dai X, et al. Co-delivery of bee venom melittin and a photosensitizer with an organic-inorganic hybrid nanocarrier for photodynamic therapy and immunotherapy. ACS Nano. 2019;13:12638-52.

42. Deng H, Zhou Z, Yang W, Lin LS, Wang S, Niu G, et al. Endoplasmic reticulum targeting to amplify immunogenic cell death for cancer immunotherapy. Nano Lett. 2020;20:1928-33.

43. Guedan S, Ruella M, June CH. Emerging cellular therapies for cancer. Annu Rev Immunol. 2019:37:145-71.

\section{Publisher's Note}

Springer Nature remains neutral with regard to jurisdictional claims in published maps and institutional affiliations.
Ready to submit your research? Choose BMC and benefit from:

- fast, convenient online submission

- thorough peer review by experienced researchers in your field

- rapid publication on acceptance

- support for research data, including large and complex data types

- gold Open Access which fosters wider collaboration and increased citations

- maximum visibility for your research: over 100M website views per year

At BMC, research is always in progress.

Learn more biomedcentral.com/submissions 